\title{
System-Level Design of GPU-Based Embedded Systems
}

\author{
Arian Maghazeh
}

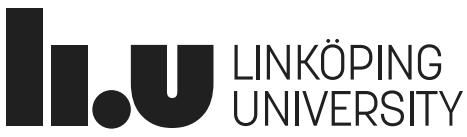

Department of Computer and Information Science

Linköping University

SE-581 83 Linköping, Sweden

Linköping 2018 
(C) 2018 Arian Maghazeh

ISBN 978-91-7685-175-3

ISSN $0345-7524$

URL https://urn.kb.se/resolve?urn=urn:nbn:se:liu:diva-152469

Printed by LiU-Tryck, Linköping 2018 
To my loved ones 



\begin{abstract}
Modern embedded systems deploy several hardware accelerators, in a heterogeneous manner, to deliver high-performance computing. Among such devices, graphics processing units (GPUs) have earned a prominent position by virtue of their immense computing power. However, a system design that relies on the sheer throughput of GPUs is often incapable of satisfying the strict power- and time-related constraints faced by the embedded systems.

This thesis presents several system-level software techniques to optimize the design of GPU-based embedded systems under various graphics and nongraphics applications. As compared to the conventional application-level optimizations, the system-wide view of our proposed techniques brings about several advantages: First, it allows for fully incorporating the limitations and requirements of the various system parts in the design process. Second, it can unveil optimization opportunities by exposing the information flow between the processing components. Third, the techniques are generally applicable to a wide range of applications with similar characteristics. In addition, multiple system-level techniques can be combined together or with the applicationlevel techniques to further improve performance.

We begin by studying some of the unique attributes of GPU-based embedded systems and discussing several factors that distinguish the design of these systems from that of the conventional high-end GPU-based systems. We then proceed to develop two techniques that address an important challenge in the design of GPU-based embedded systems, from two different perspectives. The challenge arises from the fact that GPUs require a large amount of workload to be present at runtime in order to deliver a high throughput. However, for some embedded applications, collecting large batches of input data requires an unacceptable waiting time, prompting a trade-off between throughput and latency. We also develop an optimization technique for GPU-based applications to address the memory bottleneck issue by utilizing the GPU L2 cache to shorten data access time. Moreover, in the area of graphics applications, with a focus on mobile games, we propose a power management scheme to reduce the GPU power consumption by dynamically adjusting the display resolution while considering the user's visual perception at various resolutions. We also discuss the collective impact of the proposed techniques in tackling the design challenges of the emerging complex systems.

The proposed techniques are assessed by real-life experimentations on GPU-based hardware platforms, which demonstrate the superior performance of our approaches as compared to the state-of-the-art techniques.
\end{abstract}

The research presented in this thesis has been partially funded by the National Computer Science Graduate School (cUGs) in Sweden. 



\section{Populärvetenskaplig SAMmanfattning}

En grafikprocessor (GPU) är en specialiserad datorenhet som ursprungligen utformades för att accelerera komplexa grafikrelaterade beräkningar, vilka krävs för att skapa bildpixlar som ska visas på datorskärmen. För att uppfylla denna beräkningsmässigt tunga uppgift är GPU:er utrustade med många kärnor som körs parallellt, där varje kärna arbetar på en enda pixel och där en hierarkisk minnesstruktur och hög bandbredd snabbt förser kärnorna med ingångsdata. Jämfört med kärnorna i en centralprocessor (CPU) är GPU-kärnor mindre och enklare. De arbetar dessutom på en lägre frekvens för att hålla strömförbrukningen inom ett tolerabelt spann. Därför är enkeltrådad prestanda för GPU:er lägre än för CPU:er. Tack vare det stora antalet kärnor som körs parallellt, ger GPU:er en mycket högre genomströmning. Som en analogi kan en GPU betraktas som en lång motorväg med många banor och en CPU som en kort gata.

I början av 2000-talet, då man upptäckt GPU: s potential för högpresterande datorer, satte forskarna som mål att använda GPU som beräkningsplattformar för att köra vetenskapliga applikationer som inte var relaterade till grafik. Med detta som grund inleddes ett nytt datorparadigm, nämligen generell användning av data på GPU (GPGPU). Sedan dess har många applikationer upplevt en betydande prestationsökning genom att avlasta sin massivt parallella arbetsbelastning till GPU. Exempel innefattar tillämpningar inom artificiell intelligens, video / bildbehandling, medicinsk bildbehandling, vetenskaplig databehandling och kryptovaluta. Initialt var användningen av GPGPU endast begränsad till avancerade GPU: er. Omkring år 2013 började inbyggda system dock långsamt anpassa tekniken till ett sätt att ta itu med den ökande efterfrågan på datorkapacitet. Förutom de uppenbara prestandafördelarna kan användningen av GPU i inbyggda system hjälpa till att förenkla hårdvarudesignen och minska kostnaderna genom att byta ut olika enheter på chipet (som FPGA, DSP och ASIC) samt tillhandahålla en enhetlig programmeringsram. Men för att få ut det mesta av dessa fördelar måste stora utmaningar lösas. Exempelvis är inbyggda system ofta utsatta för flera begränsningar (inklusive genomströmnings-, ström- och tidsrelaterade begränsningar) och att uppfylla alla dessa begränsningar kräver en noggrann systemkonstruktion på hög nivå.

Med GPU:ernas potential i högpresterande datorer och de unika egenskaperna hos inbyggda system som bakgrund är den centrala frågan i denna avhandling hur man optimerar prestanda för ett GPU-baserat system under en, eller en uppsättning av applikationer. Vi svarar på den frågan genom att presentera flera mjukvarutekniker på systemnivå för att optimera utformningen av GPU-baserade inbyggda system under olika grafik- och icke-grafiska applikationer. I jämförelse med de konventionella optimeringarna på applikationsnivå ger vår systemövergripande teknik flera fördelar: För det första möjliggör man att helt och hållet beakta begränsningarna och kraven för de olika systemdelarna i designprocessen. För det andra kan det avslöja optimerings- 
möjligheter genom att exponera informationsflödet mellan processkomponenterna. För det tredje är teknikerna vanligtvis tillämpliga på ett brett spektrum av tillämpningar med liknande egenskaper. Dessutom kan flera systemnivåtekniker kombineras tillsammans eller med applikationsnivåtekniker för att ytterligare förbättra prestanda.

Vi börjar med att studera några av de unika egenskaperna hos GPUbaserade inbyggda system och diskutera flera faktorer som särskiljer utformningen av dessa system från de konventionella avancerade GPU-baserade systemen. Vi fortsätter sedan med att utveckla två tekniker som tar upp en viktig utmaning i utformningen av GPU-baserade inbyggda system från olika perspektiv. Utmaningen härrör från det faktum att GPU kräver en stor mängd data för att vara närvarande vid körning för att kunna leverera en hög genomströmning. För vissa inbyggda applikationer kräver dock insamling av stora partier av data en oacceptabel väntetid, vilket kräver att man byter mellan genomströmning och latens. Vi utvecklar också en optimeringsteknik för GPUbaserade applikationer genom att använda GPU-cacheminnet för att förkorta dataåtkomsttiden. Vidare föreslår vi inom ramen för grafikapplikationer, och särskilt med fokus på mobilspel, ett energihanteringsschema för att minska GPU-strömförbrukningen genom att dynamiskt anpassa bildskärmsupplösningen, samtidigt som användarens visuella uppfattning beaktas vid olika upplösningar. Vi diskuterar också de samlade effekterna av de föreslagna teknikerna för att ta itu med designutmaningarna i nya komplexa system.

De föreslagna teknikerna utvärderas genom experiment i realtid på GPUbaserade hårdvaruplattformar, vilket visar överlägsen prestanda i våra metoder jämfört med de senaste teknikerna. 


\section{Acknowledgments}

First and foremost, I would like to thank Professor Zebo Peng, Professor Petru Eles, and Dr. Unmesh Bordoloi for their excellent support and thoughtful supervision of my Ph.D. studies and for their patience and understanding in leading me through the numerous obstacles I have been facing during these years. The things I have learned from you will always be with me.

I am grateful to Assistant Professor Sudipta Chattopadhyay for providing me the opportunity to experience a different and pleasant research environment during my academic visit to the Singapore University of Technology and Design (SUTD). I have long looked up to Sudipta and deeply admired him for his generosity, technical competence, and determination. I am also especially grateful to Associate Professor Ahmed Rezine for our inspiring discussions and for always encouraging me to stay positive.

I would like to thank Anne Moe for compassionately supporting me and all the Ph.D. students through the administrative process, from the very first day to the very last. My sincere thanks to Eva Pelayo Danils, Åsa Kärrman, Mikaela Holmbäck, Lene Rosell, and other members of the administrative staff for their constant assistance and commitment to providing a smooth and efficient working environment for all of us in ESLAB and IDA.

During my studies, I had the privilege to receive advice from several experts that helped me out of desperate situations, which every Ph.D. student occasionally encounters. I would like to express my gratitude to Professor Mattias Villani, Associate Professor Niklas Carlsson, Dr. David Byers, and Dr. Stefan Gustavson for generously providing their expert opinions when most needed.

Special thanks to my fellow labmates, from the past to the present, for their constructive feedback, cooperation, and most importantly for their great friendship; I could not have asked for better colleagues. I would also like to 
thank my other colleagues and friends in IDA for their valuable friendship. I hope our friendship will continue.

I am profoundly grateful to Professor Nahid Shahmehri and Professor Mariam Kamkar for being present in every aspect of my life during these past years and walking me through all manner of difficulties with paramount care and affection. I am forever in your debt.

My heartfelt thanks to my dearest Mikaela, who has always been beside me in the past two years through the toughest times and has given me the utmost support; thank you for your love. Finally, I would like to thank my family from the bottom of my heart for their unconditional love and support; my brother Ali, my father Farrokh, and my mother Afsaneh-I love and miss you forever.

Arian Maghazeh

Linköping, November 2018 


\section{Table of Contents}

Abstract v v

Acknowledgments $\quad$ ix

\section{PART I Thesis Summary}

1 Introduction 3

1.1 A Quick Review on GPU Computing . . . . . . . . . . . . . . . . . 3

1.2 Motivations and Objectives ............. 5

1.2.1 Potentials and Challenges of Embedded GPGPU . . . 7

1.2.2 Latency vs Throughput Dilemma . . . . . . . . . . 7

1.2.3 Managing Graphics Workload on GPUs . . . . . . . . 8

1.2.4 Addressing Memory Performance Bottleneck . . . . . 8

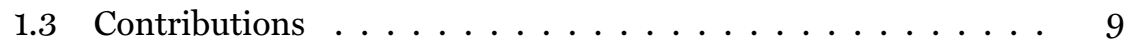

1.4 Thesis Outline ........................ 12

2 Background 13

2.1 Multi-Core CPUs vs Many-Core GPUs . . . . . . . . . . . . . . . . . 14

2.2 GPU Architecture . . . . . . . . . . . . . . 16

2.2.1 Compute Units . . . . . . . . . . . . . . 16

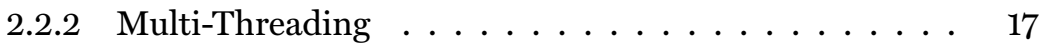

2.2 .3 Memory System . . . . . . . . . . . . . . . 18

2.3 GPU Programming . . . . . . . . . . . . . . . . . . . . 19

2.3.1 Problem Decomposition ............. 19

2.3.2 Thread Communication ............. 21

2.3.3 GPU Implementation of Matrix Multiplication . . . . 22 
2.4 Performance Considerations . . . . . . . . . . . . 24

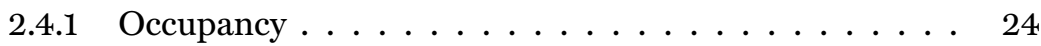

2.4 .2 Memory Latency . . . . . . . . . . . . . . 25

2.4 .3 Branch Divergence . . . . . . . . . . . . . . . 25

2.4.4 Synchronization . . . . . . . . . . . . 26

2.4 .5 Unified Virtual Memory . . . . . . . . . . . . . . . . 26

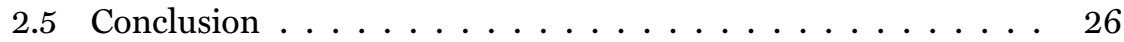

3 Related Work 29

3.1 GPGPU on Low-Power Embedded Systems $\ldots \ldots \ldots \ldots$

3.2 Real-Time Processing on Embedded GPUs . . . . . . . . . . . . 30

3.3 Power Management in Mobile Games . . . . . . . . . . . . . . 32

3.4 Packet Processing on GPUs . . . . . . . . . . . . . 33

3.5 Addressing the Memory Bottleneck . . . . . . . . . . . . . 34

4 Contributions 37

4.1 Publication List $\ldots \ldots \ldots \ldots \ldots \ldots \ldots \ldots$

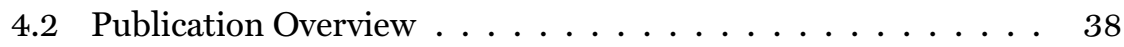

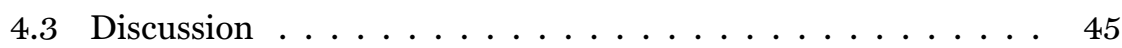

5 Conclusions and Future Work 49

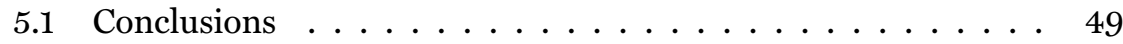

5.2 Future Work . . . . . . . . . . . . . . . . 51

$\begin{array}{ll}\text { Bibliography } & 53\end{array}$

\section{PART II Publications}

Paper I General Purpose Computing on Low-Power Embedded GPUs: Has It Come of Age?

Paper II Saving Energy Without Defying Deadlines on Mobile GPU-Based Heterogeneous Systems

Paper III Perception-Aware Power Management for Mobile Games via Dynamic Resolution Scaling

Paper IV Latency-Aware Packet Processing on CPU-GPU Heterogeneous Systems

Paper V Cache-Aware Kernel Tiling: An Approach for System-Level Performance Optimization of GPU-Based Applications 


\section{Part I}

\section{Thesis Summary}





\section{Introduction}

Today, graphics processing units (GPUs) have become an inextricable part of virtually every modern high-performance computing system. GPUs are purpose-built devices originally designed to accelerate graphics rendering. Since a decade ago, however, GPUs have been extensively used on high-end computer systems to perform general-purpose computing across various domains of applications - thanks to their enormous computing power. Lately, with the proliferation of high-performance embedded systems, GPUs quickly paved their way into the design of embedded systems. Today, a typical lowpower embedded system featuring a GPU can run algorithms that decades ago would require a large quantity of top-tier CPUs. At the same time, the emerging complex systems, such as autonomous vehicles, request for staggering amounts of processing power. This insatiable need for throughput, coupled with strict power- and time-related constraints, makes the software design of embedded systems a challenging task, worthy of in-depth study.

This thesis aims to contribute to the software design of GPU-based embedded systems. It explores some of the unique characteristics and requirements of these systems and provides several system-level techniques. The thesis also offers insights into the application scenarios of the upcoming complex systems and demonstrates how our proposed techniques can facilitate the system design. In this chapter, we give a short introduction on GPU computing, summarize the motivations and objectives, and outline the contributions of the thesis.

\subsection{A Quick Review on GPU Computing}

GPUs are specialized computing devices originally designed to accelerate complex graphics rendering calculations. A GPU typically comprises a large number of processing cores each responsible for manipulating a single pixel of an 
image frame intended to be displayed on the screen. To keep the power consumption of a GPU within a tolerable limit, the cores run at a lower clock frequency than a CPU. Despite a lower frequency, however, a typical GPU delivers a much higher throughput than its CPU counterpart, owing to (a) a pool of cores operating on different pixels in parallel, and (b) a high-bandwidth memory system, as compared to the low-bandwidth CPU memory. The highthroughput characteristic of the GPU gave rise to a new model of computing.

In the early 21st century, having discovered the potential of GPUs for high-performance computing ${ }^{1}$, some vigilant researchers set out to use GPUs as computing platforms to run scientific applications unrelated to graphics. Hence, a new computing paradigm was born, namely general-purpose computing on GPUs (GPGPU). The early GPGPU programs had to represent the problems in terms of graphics primitives that GPUs could understand. This cumbersome transformation was later resolved with the advent of generalpurpose programming interfaces, such as CUDA [8] and OpenCL [9], as these frameworks disengage the programmer from the underlying graphics concepts. In a typical workflow of a GPGPU program, the following steps take place: (a) the host (CPU) transfers the data to the GPU memory; (b) the GPU processes the data, freeing up the CPU to take care of the more sequential and control-oriented segments of the code; (c) the CPU initiates a read-back process to transfer the results from the GPU memory to the host memory.

At the time when the slowdown of Moore's law and the breakdown of Dennard scaling obstructed the steady upward trend in the single-thread performance, the emergence of GPGPU brought a renaissance to the world of computing. Soon, many applications from a wide range of domains used the GPGPU concepts to offload their massively parallel workload to the GPU. Examples include applications in the fields of video/image processing [10, 11], medical imaging [12, 13], financial modeling [14], scientific computing [15, 16], and cryptocurrency [17]. GPGPU has also sparked a revolution in the field of artificial intelligence. AI technologies like neural networks owe their remarkable achievements to the massive data-crunching capabilities of GPUs $[18,19]$. Following the success of GPGPU in high-performance computing, the next major leap happened in the area of embedded systems.

Around the year 2013, embedded systems began to slowly adopt the GPGPU technology to address the increasing demand for computing capability. In a typical CPU-GPU heterogeneous platform, the CPU handles the serial segments of the code while the GPU deals with the parallel portions. Apart from the apparent performance benefits, the use of GPUs in embedded systems can help simplify hardware design and reduce cost by replacing various devices on the chip (like FPGAs, DSPs, and ASICs). In addition, the unified C-based programming framework (or other high-level language frameworks like Python) of GPGPU facilitates the software design and reduces the devel-

\footnotetext{
${ }^{1}$ We use the term high-performance GPU computing to refer to GPGPU on high-end GPUs.
} 
opment time, as opposed to the multi-language design of a mixed-hardware architecture. On the downside, however, the general-purpose design of the GPU and its throughput-oriented characteristics may lead to power- and latencyrelated issues - two crucial performance metrics in embedded systems. The problem becomes more acute in the context of the modern complex systems with the increasing reliance on embedded computing.

In the following, we elaborate on some of the exclusive challenges that GPU-based embedded systems must contend with and explain why the current research on high-performance GPU computing alone is not enough to circumvent these challenges. We then propose several novel system-level approaches to address these issues.

\subsection{Motivations and Objectives}

Since the emergence of GPU computing, the field of GPGPU has received considerable attention from the scientific community. The great majority of the works in the GPU research focus on improving a performance-related aspect. These include advances in the fields of algorithms and applications, programming frameworks, performance analysis tools, and architecture. Studies on algorithms and applications have mostly been carried out with the capabilities of a high-end GPU in mind and the goal of increasing application throughput. The research on programming frameworks and tools aims to facilitate the design process and help identify the bottlenecks of the system. Studies on hardware architecture provide better design for different parts of the GPU and its memory system-these works can offer great performance improvements, but they are often costly and hard to deploy. The current body of research has played a major role in advancing the GPU technology and bringing it to its present status. However, considering the increasing prevalence of GPU-based embedded systems in today's technology, we believe that further research should be conducted in this area. This thesis attempts to contribute to this cause by focusing on the applications that are specific to embedded systems, taking into account the various constraints of these systems.

Embedded GPUs are used for various graphics applications, such as mobile gaming and user interfaces, as well as non-graphics applications, such as packet processing, computer vision, image processing, and neural networks. While serving different functionalities, the embedded applications often share general attributes. One common attribute is that the system input can be modeled by one or more data streams, where each data instance, henceforth referred to as an item, must go through a single or several GPU functions (where a function is a shader in the graphics context and it is a kernel in the GPU computing terminology). ${ }^{1}$ For example, in the case of mobile gaming, an item is a set of coordinates and attributes required to generate the next image frame;

\footnotetext{
${ }^{1}$ Depending on the context, we may use the notion of input to refer to a single item.
} 
in the case of packet processing, an item represents a packet; and regarding image processing and computer vision, an item is an image frame. Another attribute is that the items of a stream may arrive at a fixed or dynamic rate. For example, the arrival rate of a stream of packets, in a packet processing application, may change over time, whereas a stream coming from a camera may have a constant frame rate. Moreover, every item, regardless of the arrival rate and the stream it belongs to, is associated with a notion of timeliness. The exact realization of timeliness, however, may vary among applications: In mobile games, each output frame must be rendered within a fixed interval, which is equal to the inter-arrival time of the frames (i.e., period). Conversely, for image processing and computer vision applications, deadlines may be larger than the inter-arrival time. In a less rigid manner, timeliness may refer to minimizing the average latency per item, e.g., the average latency per packet for a packet processing application. As a result of the discrepancies in attributes, it is likely that different applications require different treatments in order to achieve the same objective. For example, to reduce energy consumption in a packet processing application, it is possible to collect and process multiple items in one batch and thereby increase the throughput per watt. The same approach cannot be applied to the graphics applications since the deadline is equal to the period. However, in the latter case, it is possible to reduce the frame size in order to reduce the amount of work per frame and hence reduce energy.

Another aspect that further makes the design of embedded GPGPU difficult is that the embedded systems are usually faced with multiple sources of concern, such as latency-, throughput-, and power-related issues. During the design phase, these concerns present themselves as either constraints or conflicting objectives. For example, accumulating multiple items may be a way to increase the energy efficiency of the GPU, but at the same time, it increases the average latency per item. Or in the case of graphics rendering, reducing the frame size lowers the energy consumption, but on the other hand, it results in a lower image quality. This degree of entanglement in constraints, however, does not appear in high-performance GPU computing. First, most high-end GPGPU applications do not have a per-item latency requirement, rather they aim to reduce the overall application duration. Moreover, although power consumption is an important performance metric in high-performance computing, it is not as much a limiting factor as it is in the embedded system designdue to the access of high-performance systems to the continuous power supply.

The above examples illustrate some of the complexities in GPU-based embedded systems and underscore the importance of design optimization. To overcome these challenges, we believe that it is required to optimize the design both at the low level and high level. A low-level design deals with optimizing performance with respect to a particular system component (e.g., a processing node). At this level of abstraction, the main limitation is that the design is oblivious to the requirements of the rest of the system. For example, a packet processing application that is solely optimized to maximize throughput 
requires to collect a large batch of packets before launching the GPU kernel. But, if the arrival rate is low, this approach results in a larger packet latency (mainly due to a larger waiting time to build the batch). On the other hand, a system-level view would allow for monitoring the arrival rate and setting the throughput according to the current arrival rate. In addition, a high-level design may expose optimization opportunities that are often not visible at the component level. For example, by exploiting the flow of information and the interrelation between the components, a high-level design is able to accelerate data transfer between processing nodes. Finally, such a design may also be used in system configurations where the GPU is shared by several applications. In these scenarios, a high-level view is needed to efficiently utilize the GPU while considering the requirements of all applications.

Below, we enumerate the main objectives pursued in this thesis and discuss their significance.

\subsubsection{Potentials and Challenges of Embedded GPGPU}

As an early step into our research, we started off by characterizing some of the potentials and challenges of embedded GPGPU, at the time when very little research had been conducted in this area. This was crucial because the common high-performance GPGPU concepts do not readily fit the requirements of the embedded systems, due to the architectural and operational differences.

\subsubsection{Latency vs Throughput Dilemma}

GPUs are throughput-oriented machines, but they require large volumes of data to deliver a high throughput. This is to keep the GPU pipeline busy with ready instructions while others are waiting for the results of long-latency operations. Most research studies that involve optimization techniques presume a large input size adequate to keep the GPU(s) busy during the kernel execution, where the input represents a single large item or a collection of small items. In the latter case, the entire batch is regarded as a single task and the applied optimization works to reduce the execution time of the aggregate task. The input streams in some embedded applications have similar properties in that the workload of a single item is not enough to attain a high device utilization. Hence, batching is required. However, there is a problem with this approach: the embedded applications often have real-time requirements, and as such the waiting time required to build the batch can lead to the violation of the timing constraints. The problem aggravates when we consider that as GPUs become more powerful, the minimum number of items required to reach the GPU's peak throughput (for a given task) increases. Consequently, the minimum waiting delay also increases, possibly resulting in a higher latency per single item-even though the processing delay of a single item may have been lower due to a better design of the GPU pipeline. Therefore, a careful system-level 
design is required that, on one hand, strives to improve the GPU utilization (and hence the throughput and energy efficiency of the system) by forming reasonably large batches, and on the other hand, makes sure that the timing requirements are satisfied.

Even though several embedded applications essentially face the same latency vs throughput dilemma, the exact characteristics and requirements of their system models may differ. For example, in one variety of applications, the items from various streams may need to go through an identical function and be processed within a predetermined interval. In this case, given a fixed number of streams with fixed arrival rates, the objective can be defined as to produce a schedule that minimizes the energy consumption. In another scenario, the minimum required throughput of the system may change over time, due to the dynamic arrival rate of the input stream. In this case, the objective may be to minimize the average delay per item while meeting the minimum required throughput at all time.

\subsubsection{Managing Graphics Workload on GPUs}

In graphics applications, image frames must be rendered by the GPU before a certain deadline. For example, the frames of a video game that runs at $60 \mathrm{~Hz}$ must be processed within 16 milliseconds. For each frame, the amount of work that the GPU must undertake depends on the number of pixels in the frame, i.e., the frame resolution. As mobile screens have continuously grown in size and resolution, the energy consumption per frame has significantly increased. However, this supposed improvement in image quality does not always justify the implications of a higher GPU power, e.g., a shorter battery life. To put it another way, users may be willing to sacrifice the image quality to a certain extent in order to extend the battery life of a mobile device. Therefore, it is desirable to be able to change the resolution of graphics applications to reduce power consumption. Moreover, as users have different perceptual sensitivities to frame resolution, it is also important that such an approach integrates the preferences of the particular user in various conditions, in order to provide the best user experience for the longest time. This technique may also be used in the cases where the GPU is shared between several graphics and non-graphics applications, in order to reduce the GPU workload so that it can provide better service to more applications.

\subsubsection{Addressing Memory Performance Bottleneck}

Despite GPU's ability to hide memory latency, memory access time is the principal performance bottleneck in GPGPU applications. Prior studies on this issue involve both hardware and software approaches. Hardware techniques primarily focus on (a) developing faster memory technologies and communication links, and (b) architectural innovations like a better cache design. Soft- 
ware optimizations that deal with memory-related issues are chiefly based on exploiting the data locality that is embedded in the application. Spatial locality is used to reduce the number of memory transactions, as in memory coalescing techniques. Temporal locality is exploited to store a small amount of data in high-speed on-chip memories (like scratchpad and cache memories) for faster future accesses.

In temporal-locality-based techniques, the data is first fetched from the slow off-chip memory and then stored in a high-speed on-chip memory to accelerate further accesses. The latency of the first access to the data, however, may substantially affect the performance of the kernels with low temporal locality. In such kernels, the first data access may contribute largely to the overall time spent on accessing the element and processing it. Therefore, eliminating the first-access latency can help improve the performance. One possible way to attack this problem is via using the GPU L2 cache. Every access to the global memory first goes through the L2 cache and is responded if the data is found in the cache. By ensuring that upon the first access the data resides in the cache, we can eliminate the slow access to the global memory and potentially improve the performance of the application. Another advantage of this technique is that, unlike traditional techniques, which are dependent on the inner-workings of the application, it is generally applicable to any kernel as long as it satisfies certain conditions. However, one major challenge involves making sure that the data element that is going to be accessed soon will not be evicted from the cache by competing memory accesses. This is not trivial because, unlike software-controlled scratchpad memories, caches are automatically managed by the hardware and as such the designer has no explicit control over the cache content.

\subsection{Contributions}

The main contributions of this thesis are in the area of system-level design techniques to improve the performance of GPU-based embedded systems. We contribute towards identifying some of the distinct challenges in the high-level software design of these systems, and we propose techniques to address these challenges under graphics and non-graphics streaming applications. In the following, we outline the main contributions made in the thesis. Each contribution corresponds to one of the included papers, ordered by the time of publication.

\section{Paper I. Exploring the potential of GPUs for energy-efficient computing in low-power embedded systems. Examine the opportunities and challenges in the design of embedded GPGPU applications.}

- We present one of the earliest works to study the potential of embedded GPUs for high-performance energy-efficient computing by 
implementing five non-graphics benchmarks on a low-power platform with GPGPU support.

- We empirically demonstrate that simply porting a kernel optimized according to the features of a high-end GPU onto an embedded GPU, without considering the architectural limitations of these devices, often results in a non-optimal performance.

- We compare the execution time and energy consumption of several applications on the GPU with those on the single, dual, and quad CPU cores and show early promising results indicating that in the future the embedded systems may be able to run applications that historically perceived to be computationally infeasible to host.

Paper II. Exploiting GPUs for energy-efficient computing in real-time systems. Propose a scheduling mechanism for time-constrained data streams on CPU-GPU heterogeneous platforms.

- The same throughput-oriented characteristic of GPUs that serve well the high-throughput requirements of real-time systems presents major challenges in satisfying their timeliness requirements. We propose a scheduling scheme to utilize both the GPU and CPU harmoniously to meet the deadlines of a real-time application while maximizing energy savings.

- We propose a fast near-optimal heuristic to map the items of the input streams onto either of the GPU or CPU cores and schedule the execution of jobs on each processor. At the core of the proposed iterative heuristic is an efficient algorithm that solves the scheduling problem by converting it into a problem of finding the shortest path in a graph.

- We evaluate the energy efficiency of our proposed technique by conducting experiments on a hardware platform and present significant energy savings.

Paper III. Reducing power consumption on mobile devices by lowering display resolution. Propose a mechanism to extend battery life via dynamic resolution scaling while considering user perception.

- We empirically demonstrate that pixel rendering on GPUs contributes remarkably to the overall system power of mobile devices. We provide a lightweight knob in the Android framework to enable dynamic resolution scaling for closed-source Android games.

- We propose a power management mechanism that exploits the knob to automatically adjust display resolution at runtime in accordance with user preferences. The scheme is based on a statisti- 
cal model that captures user preferences and at runtime predicts the user's choice according to the current state of the system.

- We also propose an accurate GPU power model to estimate the GPU power consumption at runtime based on the current utilization of the device and its operating frequency. We build a similar power model for the memory unit.

Paper IV. Latency-aware packet processing on low-power GPU-based heterogeneous platforms. Propose a new perspective on packet processing on GPUs using persistent kernels.

- While processing packets in fixed-size large batches favors the GPU throughput, it imposes additional packet latency, particularly when the arrival rate is low. We propose an adaptive technique to change the batch size dynamically according to the traffic rate. To this end, we propose the use of a persistent kernel that is launched only once and continuously processes the batches of different sizes.

- We propose a software architecture that exploits the unified memory architecture in the heterogeneous platforms to efficiently coordinate the exchanging of packets between the CPU and GPU, and seamlessly communicate the new batch sizes to the GPU.

- We use a packet classification algorithm to showcase the advantages of the proposed technique, as compared to the conventional methods, in reducing the average packet latency while maintaining a sufficiently high throughput to avoid any packet loss.

Paper V. Exploiting the GPU L2 cache to improve the performance of GPU-based applications. Propose an approach to accelerating the data transfer between data dependent kernels.

- Memory latency is a major performance bottleneck for many kernels. We demonstrate that the performance of some kernels can significantly be improved if the initial accesses to the input data are conducted via the cache, instead of the global memory. We propose three conditions to identify such kernels.

- We develop a tool to reduce the overall execution time of an application graph by splitting the cache-sensitive kernels in the graph into smaller sub-kernels so that each sub-kernel may find its input in the GPU L2 cache. We apply the tool to a full-fledged application and demonstrate significant performance improvements.

- We propose a technique to construct the data-dependency graph and obtain memory footprints for arbitrary GPU-based applications at the block level. 


\subsection{Thesis Outline}

This thesis is a compilation of five research papers, as outlined in Section 1.3. It is organized in two parts. In continuation of this first part, Chapter 2 and Chapter 3 provide a general overview of the GPU design and GPGPU concepts and survey the previous research in the area, respectively. Chapter 4 provides a summary of each paper along with a discussion on some potential future applications of the proposed techniques. Part I ends with conclusions and future work in Chapter 5.

Each paper in Part II takes a different perspective to address the main research question of the thesis, which is about improving the performance of a GPU-based embedded system using system-level software techniques. Paper I outlines some of the opportunities and challenges in embedded GPGPU design. Paper II and paper IV deal with providing a good balance between the throughput of the application and the latency per item, which is an intrinsic dilemma in the GPGPU design. Paper III focuses on traditional graphics aspect of GPUs and strives to reduce the GPU power and energy consumption in mobile games. Finally, paper $\mathrm{V}$ addresses the memory bottleneck issue by utilizing the GPU L2 cache to shorten the data access time. In Part II, each chapter is a published paper presented at a conference. 


\section{Background}

For almost four decades (from the 1970s to mid-2000s), thread performance on a single-core CPU roughly doubled every two years. During this period, the scaling of fabrication technologies, aligned with Moore's law [20], provided the chip designers with smaller and faster transistors, while Dennard scaling ensured that the power density remained practically constant [21]. The extra transistors were spent on advancing the CPU control unit and integrating larger caches, and as such the performance of sequential programs steadily improved. However, with the breakdown of Dennard scaling at around 2006, this upward trend came to an end. The reason was that as transistors continued to shrink in size, their power consumption did not drop at the same rate; therefore, to prevent the processor from overheating, the ramping up of the clock frequency had to stop. Meanwhile, fabrication technologies continued to scale according to Moore's law. Taking a new design perspective, manufacturers then focused to use the extra transistors to integrate more computing cores into the chips. The shift created a huge impact on the software developer community, who was by then accustomed to relying on advances in hardware to automatically accelerate their programs. As sequential programs could not experience major speedups anymore (due to frequency stabilization), programmers turned to concurrent programs [22] to increase the performance of their applications by utilizing the cores in parallel. The following surge towards writing parallel programs gave rise to a new era of concurrent programming.

Among devices that support parallel computing, GPUs have gained popularity, primarily thanks to their tremendous computing power, the generality of use, and the ease of programming. In this chapter, we provide background information about the GPU architecture, GPGPU concepts and programming models, and several performance considerations in GPGPU programming. 


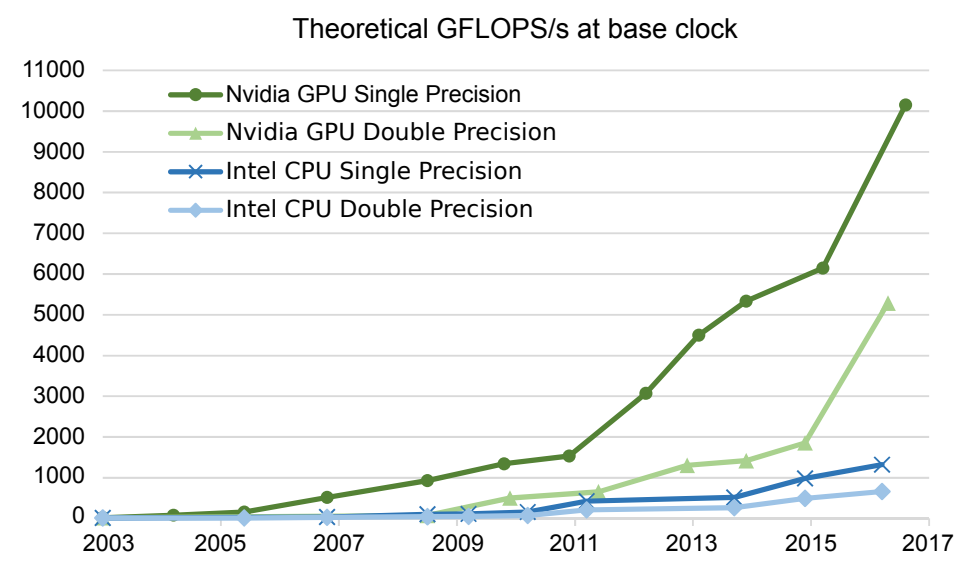

Figure 2.1: Comparing throughput gap between CPUs and GPUs (figure recreated from NVIDIA [23])

\subsection{Multi-Core CPUs vs Many-Core GPUs}

To explain the design philosophy of GPUs, we compare the many-core architecture of these devices with the multi-core architecture of today's CPUs.

Multi-core processors improve performance by allowing separate sequential programs, or different sections of the same program, to run on different cores in parallel [24]. The core architecture of a multi-core processor is similar to that of a single-core processor, in the sense that each core uses sophisticated control logic (including circuitry for out-of-order execution, multipleinstruction issue, and branch prediction) and a hierarchical cache structure to maximize the execution speed of sequential programs [25].

In contrast, GPU-like many-core architectures comprise many small cores with simple control units (e.g., with in-order execution, single-instruction issue, and no branch prediction unit), where several cores share the same control unit. GPUs also have much smaller caches than multi-core CPUs. Instead, the precious chip space is dedicated to cramming more cores onto the die. Moreover, GPU cores run at lower frequencies than CPU cores, so that more cores may be switched on simultaneously without overheating the chip/board. To supply the cores with sufficient data, GPUs rely on high-bandwidth memory modules-as opposed to faster but low-bandwidth CPU memories. As a result of these architectural discrepancies, a many-core GPU can achieve a much higher throughput than a multi-core CPU, while the performance of a single thread is higher on a CPU core [25]. As an analogy to describe the difference between the two, CPUs can be seen as short streets between two points, while GPUs are analogous to multi-lane long highways connecting the same points. 
Theoretical Peak GB/s

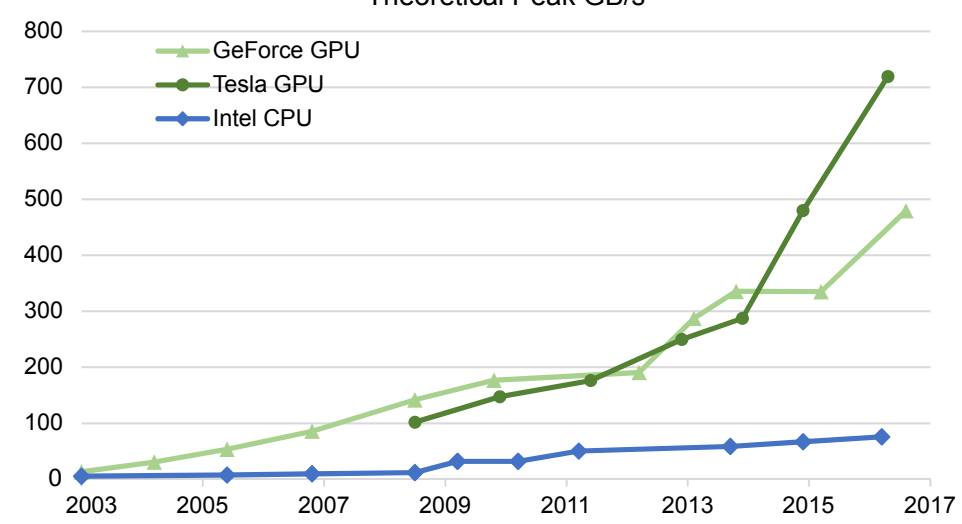

Figure 2.2: Comparing memory bandwidth between CPUs and GPUs (figure recreated from NVIDIA [23])

Figure 2.1 and Figure 2.2 illustrate the gap between the throughput and memory bandwidth of NVIDIA GPUs and Intel CPUs, respectively.

From the energy perspective, processing highly parallel data-sets on GPUs is much more energy efficient than on CPUs. In GPUs, every instruction fetch is followed by several thread executions (e.g., 16 or 32) performed on different pieces of data. Thus, the energy that is used for fetching an instruction, which is significantly larger than the energy it takes to actually execute the instruction [26], is amortized over a large number of thread executions. In comparison, a CPU must fetch the same instruction repeatedly (once per execution) in order to process the same amount of data, thereby consuming more energy.

Despite GPU's superior performance in handling highly parallel computations, the actual performance gain that an application may experience from GPU processing is limited by the amount of serial code [27]. For example, suppose that the parallel part of an application can be accelerated one hundred times on a GPU. In this case, if ninety percent of the application code must be performed serially (while the rest can run on the GPU), the overall performance gain is limited to roughly ten percent. On the other hand, if the serial segment constitutes ten percent of the application code, the application can be sped up by almost a factor of ten. This example demonstrates that while GPUs can boost the performance of some application parts, the overall performance improvement can be considerably less. In such cases, the design efforts should also be directed towards optimizing the serial code on the CPU. In fact, for many applications, the best performance comes when the CPU and GPU are used together to match the requirements of the application to the strengths of each processing unit while achieving high utilization on each device [28]. 


\subsection{GPU Architecture}

The first GPUs used fixed-function hardware to accelerate 2D and 3D rendering pipelines. In 2001, NVIDIA unveiled the first programmable GPU (namely GeForce 3), where pixels and vertices could be processed by short programs, known as shaders, to create various rendering effects [29]. Early GPUs included different types of shader hardware, e.g., vertex shaders to transform the position of each vertex and pixel shaders to compute the color and other attributes of each pixel. Soon the graphics industry evolved towards a unified shader architecture, where all shader cores use the same hardware resources to perform any type of shading [30,31]. While the primary function of the shader cores was to run shading algorithms, in principle, they were capable of running any program. This feature was exploited later to run non-graphics applications on GPUs. The unified shader model allowed for a more balanced use of the shader cores and has been ubiquitously used in GPUs since then.

\subsubsection{Compute Units}

A unified GPU device contains an array of many streaming processor (SP) cores (i.e., shader cores), typically clustered into multi-threaded streaming multiprocessors (SM). An example of a modern GPU is the giant NVIDIA TITAN V GPU with Volta micro-architecture, which contains 5120 SP cores (or CUDA cores in NVIDIA terminology) organized into 80 SMs with each SM encompassing 64 SP cores. We note that the number of SPs per SM and the number of SMs per GPU vary with GPU generation. Figure 2.3 shows a Volta streaming multiprocessor. ${ }^{1}$ Each SP core includes one 32-bit integer unit and one 32-bit (single-precision) floating-point unit, enabling simultaneous execution of INT32 and FP32 operations. Each SM also contains 32 64-bit (doubleprecision) floating-point units to execute FP64 operations and 16 SFU units, organized in four groups of four, to execute instructions that compute special functions (e.g., transcendental functions) on single-precision floating-points. These instructions can run concurrently with instructions running on the SP cores. With Volta, NVIDIA has also introduced tensor cores. These are dedicated units designed to accelerate matrix multiplication operations, which are at the core of neural network training and inferencing [32].

The cores on an SM are organized into four processing blocks, where each block executes the instructions issued by a warp scheduler unit. Each core in a block executes the same instruction for a single thread, in parallel with other cores in the block. As explained in Section 2.1, in GPUs, several threads are grouped together and execute the same instructions in a lockstep fashion. NVIDIA calls such a group of threads a warp, where each warp includes

\footnotetext{
${ }^{1}$ In this chapter, we use NVIDIA GPU architecture and terminology to explain the hardware and software aspects of GPU design. While the architectural details and terminologies vary among different GPU vendors, the underlying concepts are the same.
} 


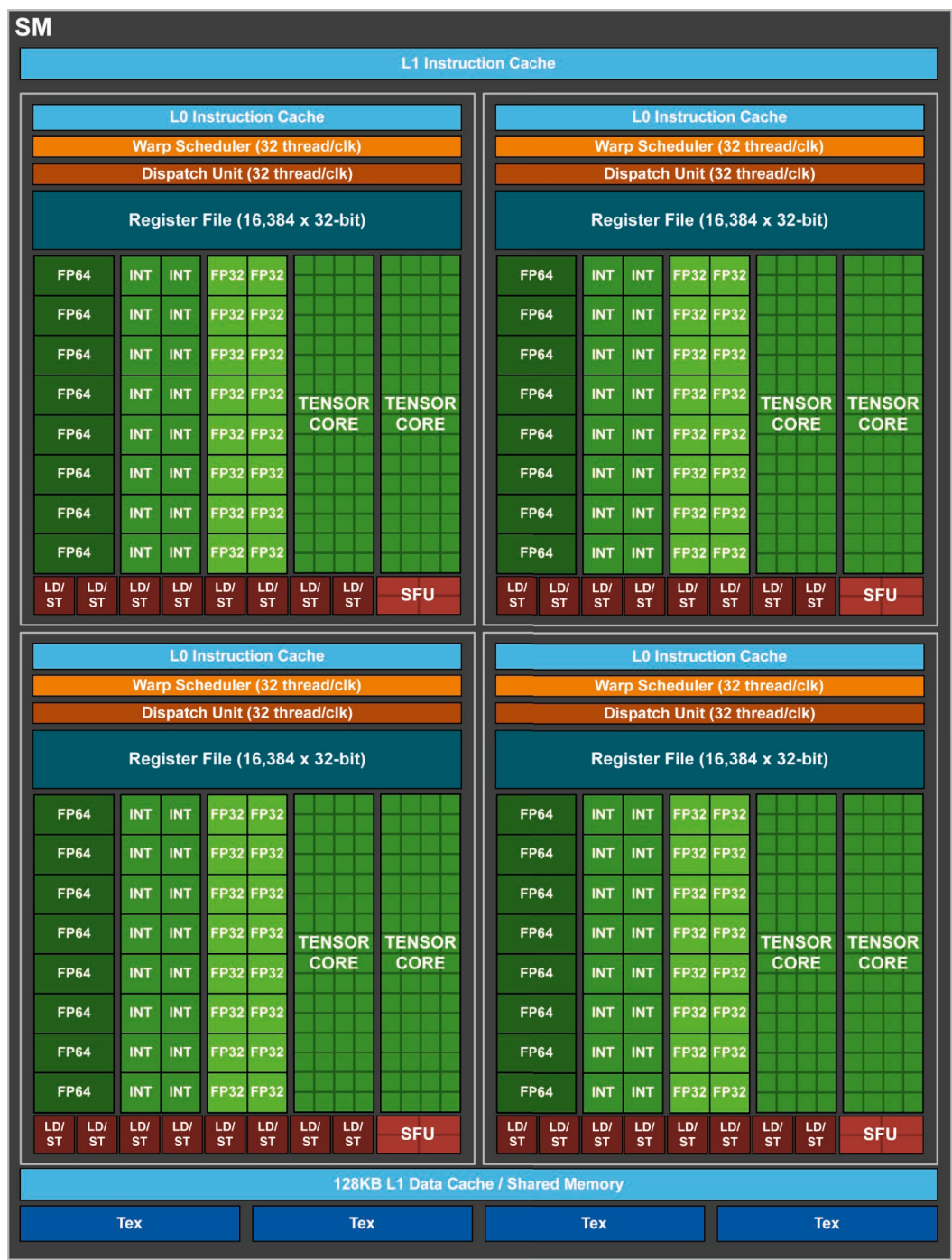

Figure 2.3: NVIDIA Volta streaming multiprocessor (courtesy of NVIDIA)

32 threads. On a Volta SM, it takes two cycles to issue an IMAD/FMAD (integer/single-precision floating-point multiply-add) instruction from a warp (with 32 threads) to one of the four processing blocks (with 16 SP cores).

\subsubsection{Multi-Threading}

The SM is heavily multi-threaded, supporting hardware resources for up to 2048 threads to be resident at any time. Note that a typical program usually 
declares thousands or millions of threads [33]. At runtime, the GPU brings in only a small fraction of the entire batch of threads by assigning them to SMs; other threads are kept in the queue waiting to be scheduled. Each SM has four large register files containing a total of $64 \times 1024$ general-purpose 32 -bit registers divided among the resident threads. The number of registers per thread depends, for the most part, on the program demand. Low register demands result in many threads (up to 2048) running concurrently on an SM, while high demands result in fewer resident threads with more registers (up to 255 registers) per thread. Deciding the exact number of registers per thread is an optimization problem that involves finding a balance between cutting down on the register usage per thread, to increase multi-threading, and the cost of register spilling, which occurs when variables are transferred from registers to DRAM memory. The register allocation is performed by the compiler. However, programmers can use compiler directives to influence the decision [34].

As an implication of such a massively multi-threaded architecture, GPUs rely on fine-grained data-parallel programs to efficiently utilize their cores. A large number of threads also helps hide the memory latency: In GPUs, caches are small and DRAM memory is slow; therefore, memory accesses often take hundreds of processor clock cycles [24]. Having many warps ready to execute allows the GPU to immediately issue an instruction from one of the ready resident warps and by doing so cover the memory latency with useful operations while the threads of the blocked warp are waiting for the results of memory accesses. GPUs can issue instructions from different warps without delay because in GPUs there is no inter-warp context switching; the execution context (program counters, registers, etc.) for each warp is stored on the chip during the lifetime of the warp, unlike traditional context switching, which requires saving registers to memory and restoring them.

\subsubsection{Memory System}

Many GPUs use a load/store architecture approach [35], where the instructions are divided into memory accesses (involving load or store between memory and registers) and ALU operations (involving only registers). Each processing block in a Volta SM includes $8 \mathrm{LD} / \mathrm{ST}$ units, where each unit handles the memory operation of a single thread. When a warp executes a memory instruction, the $\mathrm{LD} / \mathrm{ST}$ units must process 32 loads or stores. In each cycle, 8 memory addresses are calculated and coalesced into as many 32-byte contiguous memory blocks as required. For each 32-byte memory block, a memory transaction is then initiated in one cycle (as mentioned earlier, each transaction takes hundreds of clock cycles to complete). Therefore, memory accesses to adjacent addresses are coalesced together and result in fewer transactions and increased effective bandwidth. For example, a warp can access a group of 32 integers that are stored successively in the memory via four 32-byte transactions, with each transaction fulfilling the memory requests of eight threads. 
On the other hand, an entire block is fetched even if only a single byte is accessed. For example, it takes 32 transactions to access 32 integers in the memory with a minimum distance of 32 bytes between them.

GPUs use a hierarchical memory model to accelerate the distribution of data to the cores. At the lowest level, each thread can access a limited number of registers at the highest speed. Registers are private memory units that can be accessed only by the individual threads. Each SM also contains a fast onchip memory which is used partly as the shared memory (a software-managed scratchpad memory) and partly as the L1 data cache. The shared memory is evenly partitioned between groups of warps, called thread blocks (explained in Section 2.3.1). Threads within the same thread block may access the same shared memory partition. At higher levels of abstraction, there are global, local, texture and constant memory spaces, each used for a different purpose. These memory spaces reside on off-chip DRAM modules. As compared to the on-chip memory, DRAM memory is significantly larger but slower. In a highend device, the GPU has its own dedicated DRAM memory (which in turn connects to the system memory via PCI express channel), while in an embedded GPU the memory is shared with the CPU. All SMs also share an L2 cache.

\subsection{GPU Programming}

The advent of programmable shaders and floating-point support on GPUs in 2001 enabled programmers to express certain problems (e.g., those involving matrices and vectors) in terms of graphics primitives and perform them much faster on the GPU. This process was facilitated later as GPU programming frameworks, such as CUDA [8] and OpenCL [9], and high-level application programming interfaces (APIs), such as OpenMP [36], OpenACC [37] and SkePU [38], enabled the programmers to specify their inherently parallel problems using high-level languages, such as $\mathrm{C}$ and $\mathrm{C}++$. These APIs are used today to program high-end and embedded CPU-GPU heterogeneous systems.

In this section, we discuss how a data-parallel problem is partitioned so that it can efficiently be mapped to the GPU. Then, we discuss two primary mechanisms in GPUs that allow threads to communicate with each other with efficiency and reliability. Finally, a CUDA implementation of matrix multiplication is provided to illustrate some of the GPU programming concepts.

\subsubsection{Problem Decomposition}

Data-parallel computing is about solving a problem with a large data-set by decomposing it into sub-problems with smaller data-sets that can be solved in parallel. In the simplest form, every task reads from and writes to specific memory elements in a one-to-one correspondence between input and output. In this style, each task is assigned to a single thread and threads work independently to solve the problem. An example is adding two or more arrays into 
an array of sums. However, building a parallel framework based on this approach lacks flexibility because not all problems can be easily mapped in this way to the GPU. In fact, many problems exhibit a many-to-many input-output relation, where multiple input elements contribute to the value of an output element and every input element contributes to the values of multiple output elements. In this type of problems, multiple threads work together to compute an output element. In some cases, threads must cooperate in order to prevent race conditions; for example, when multiple threads update the same output element (e.g., in a histogram), or when threads read the partial results produced by other threads (e.g., in a sum reduction). Threads may also cooperate to improve performance; for example, when there is data reuse among threads, threads may collectively retrieve the required data block, by each thread reading one or more data elements from the off-chip memory once, and then share the data among each other via the on-chip memory-instead of each thread collecting all its input elements individually. An example of this problem type is a matrix multiplication, where each element of the input arrays is used multiple times to calculate the values of several output elements.

A parallel framework that supports communication among threads can be built on a two-level partitioning scheme. First, the problem is partitioned into blocks, during a coarse-grained partitioning, where each block can be processed independently in parallel with other blocks. The blocks are further partitioned into threads, during a fine-grained partitioning, where threads within a block run in parallel and cooperatively produce the results for that block. Such a problem decomposition fits particularly well to the GPU architecture; the blocks are mapped to the SMs and the threads within each block run on the SPs. Figure 2.4 shows a problem decomposition for a matrix multiplication between two $4 \times 4$ matrices. The problem is partitioned into four blocks of $2 \times 2$ threads each. Without thread cooperation, each element of the input arrays is read four times from the off-chip global memory. The two-level approach reduces this number to two, while the other two accesses are made via the on-chip shared memory. This is shown in Figure 2.4 via the four accesses to matrix element $A_{2,0}$. In general, for matrix multiplication, using $\mathrm{N} \times \mathrm{N}$ blocks can reduce the number of global memory accesses by a factor of $\mathrm{N}$.

To recapitulate, we use the CUDA terminology to define the main elements of a data-parallel framework as follows: A thread is the smallest software execution unit that represents the execution of the kernel. A kernel is a small program run by all threads on the GPU. A group of 32 threads form a warp. Threads in a warp execute instructions in a lockstep fashion. A group of warps are organized into a one-, two-, or three-dimensional block. A block is executed on an SM. Blocks execute independently from each other and are further organized into a one-, two-, or three-dimensional grid. The minimum grid size is one block. A kernel is specified by its grid size and its block size. 


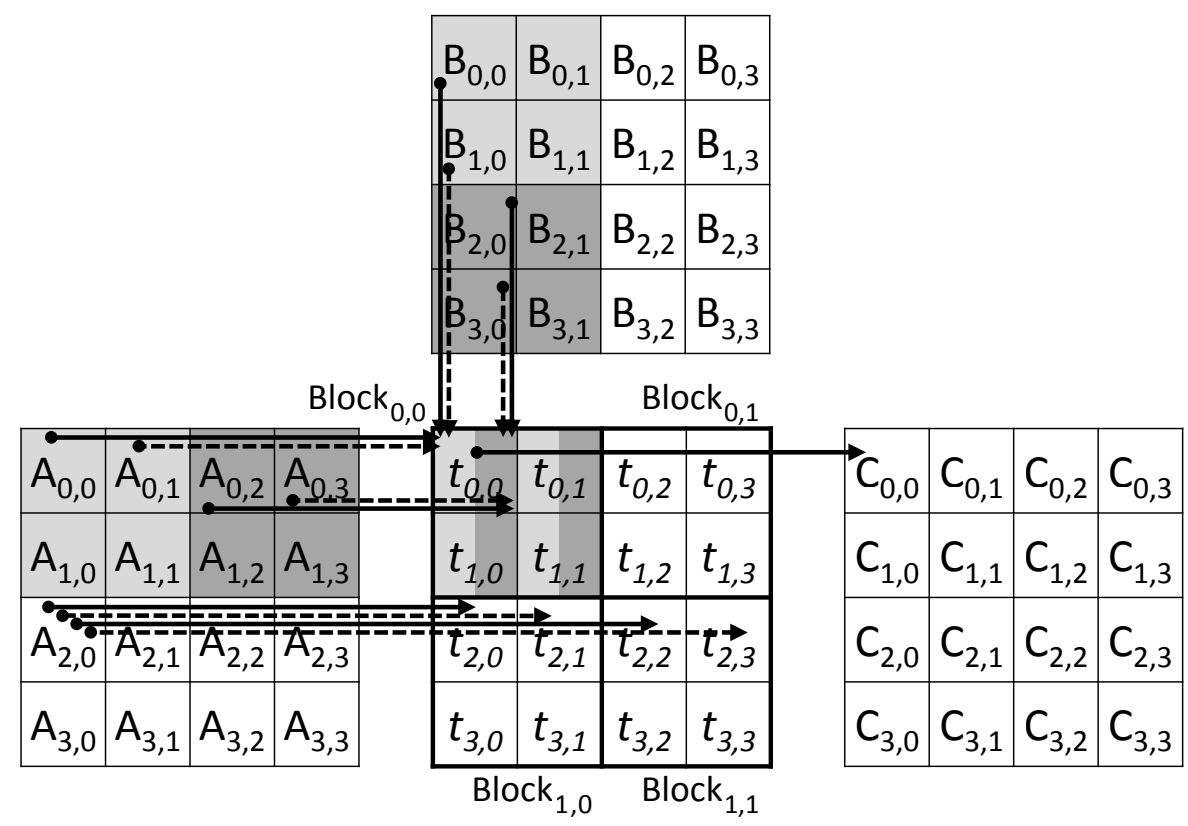

Figure 2.4: Parallel decomposition of matrix multiplication using four blocks of four threads each. Each block produces a quarter of the output matrix $\mathrm{C}$ in two steps: First, the two quarter matrices of the two inputs (i.e., A and B matrices), shaded in light gray, are multiplied. The resulting matrix is then summed up with the outcome of the multiplication between the other two input quarter matrices, shaded in dark gray. The solid and dashed arrows respectively denote the off-chip (global) and on-chip (shared) memory accesses made by a thread to a matrix element.

\subsubsection{Thread Communication}

The two primary mechanisms that enable efficient thread communication in GPUs include shared memory and thread synchronization. Shared memoryan on-chip software-controlled piece of memory partitioned among the blocks with each partition being shared by the threads within a block-allows for blocks to operate like small computing engines with access to fast on-chip memories. Through the shared memory, threads within a block may efficiently exchange data and, also, store the data with high temporal locality for fast future accesses. However, the amount of shared memory allocated to a block must be decided carefully because the total amount of shared memory per SM is limited. Therefore, overconsumption of shared memory per block results in fewer resident blocks on the SM and less parallelism. Another efficient way for thread communication is warp shuffling, which is a warp-level primitive that allows the threads within a warp to read one another's registers [39]. 
GPUs use various synchronization techniques to ensure the correct ordering of concurrent events. Among them, the most common technique is barrier synchronization, denoted as _ syncthreads() in CUDA. A barrier is a construct that is used for reliable intra-block communication. It ensures that all active threads within a block reach the same barrier instruction before any of them can progress beyond the barrier. All threads in the block must participate in the synchronization process [23]; if some threads cannot reach the barrier (for example, if the barrier is placed in a path of a branch that is not traversed by all active threads), the remaining threads will wait indefinitely at the barrier and a deadlock arises. Between thread blocks, an implicit synchronization takes place at the end of the kernel. However, with the recent Pascal and Volta architectures, NVIDIA has introduced the concept of cooperative groups, which provides synchronization among the threads in a group, and where a group can span the entire grid [40].

Other major classes of GPU synchronization techniques include memory fence functions and atomic operations. GPUs follow a weakly-ordered memory model, which means that the order in which a thread writes data to memory is not necessarily the order in which the data is observed by another GPU thread or a host thread [23]. Memory fence functions are used to enforce ordering on memory accesses. CUDA provides three fence functions to ensure ordering in the scopes of block, device, and system (i.e., multiple devices and the host). Moreover, atomic functions allow threads to perform a readmodify-write operation on a memory word (global or shared) without interference from other threads. CUDA provides several arithmetic (add, sub, min, max, etc.) and bitwise (and, or, and xor) atomic operations. Recent CUDA architectures also allow developers to define the scope of an atomic operation at the level of block, device, or system (i.e., between the CPU and GPU). CUDA also provides an atomic compare-and-swap operation, which can be used to enforce inter-block synchronization (while _ syncthreads() is used for intrablock synchronization).

\subsubsection{GPU Implementation of Matrix Multiplication}

Listing 2.1 shows a CUDA implementation of multiplication between two square matrices. On the host, the following preparatory steps take place: The size of the square-shaped thread block is determined by setting its width to a constant value $\mathrm{N}$. Each block produces an $\mathrm{N} \times \mathrm{N}$ tile of the output matrix, as explained in Section 2.3.1. The host allocates memory space on the GPU for the input and output matrices and copies the input matrices from the host to the GPU memory. The kernel is invoked by specifying the number of blocks in the grid and the number of threads in a block. The pointers to the matrices on the device and the width of the input matrices are also passed to the kernel as arguments. As execution on the GPU is non-blocking, the kernel call immediately returns. We use cudaDeviceSynchronize() function to block the host 


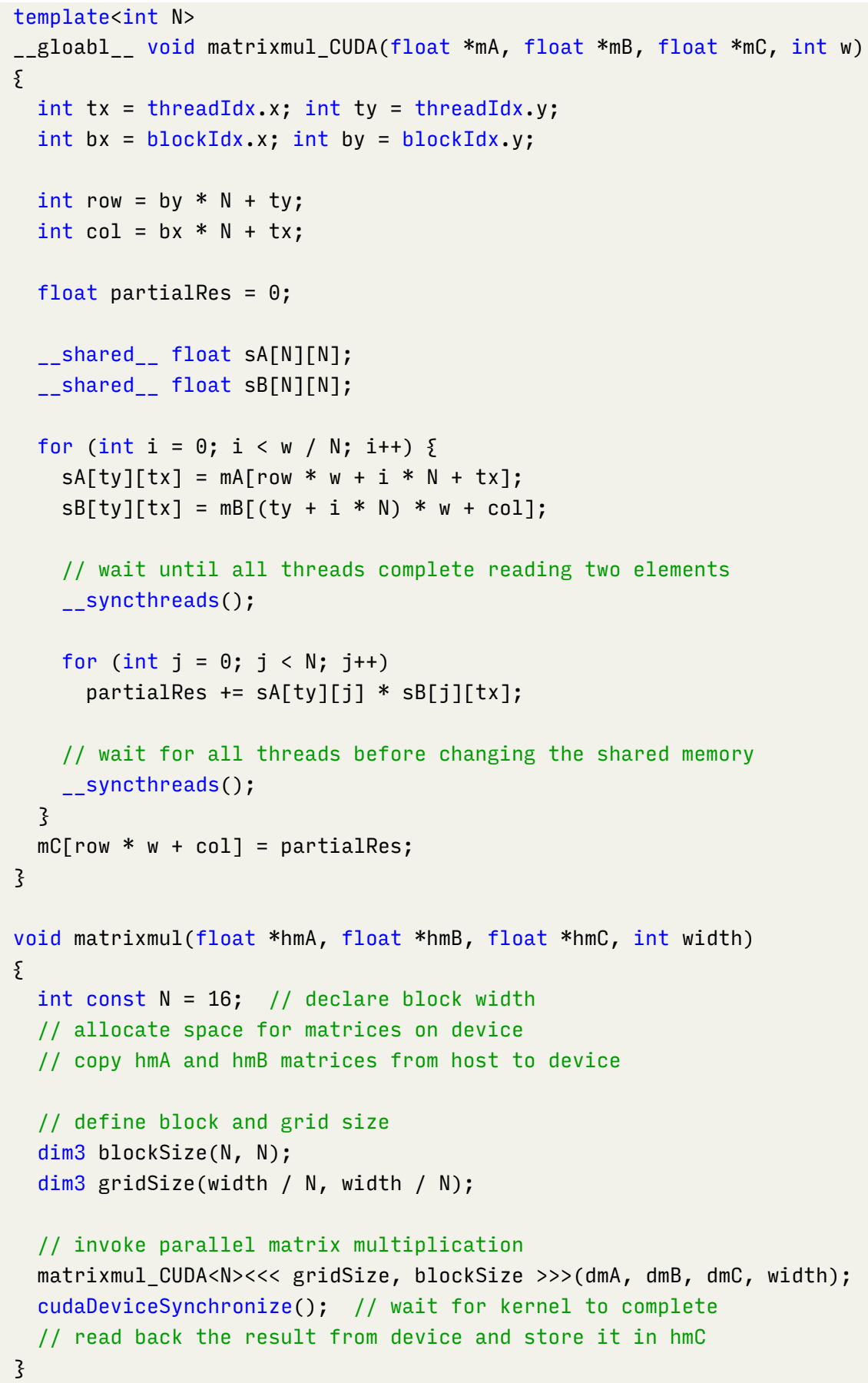

Listing 2.1: CUDA implementation of square matrix multiplication 
thread until the GPU completes its work. The output matrix is then copied from the GPU memory.

The kernel instructions specify the execution of a single thread. Initially, each thread obtains its local coordinates in the block and the coordinates of its block in the grid. A thread also calculates the id of the row in matrix A and the id of the column in matrix B, whose inner product it is going to calculate. Blocks iteratively pass over the two input matrices in units of $\mathrm{N} \times \mathrm{N}$ tiles and calculate the inner product for each pair of tiles (similar to Figure 2.4). Each block allocates two arrays of shared memory to store the data elements of the current tiles being processed. In each iteration, the threads collaboratively fetch one tile from each input matrix from the global memory and store them in the shared memory. After all threads have loaded their two elements, each calculates the inner product of its corresponding row and column of the two tiles and adds the result to the partial result that has been calculated during the previous iterations. When all tiles are traversed, each thread writes its final result into the corresponding element of the output matrix.

\subsection{Performance Considerations}

Several barriers may impede a GPU program from harnessing the true power of GPUs. For example, because GPUs dedicate most of the chip space to parallel execution pipelines, the control units are simple, and the caches are relatively small. One implication of a small control unit is that, instead of relying on hardware techniques to improve the flow in the instruction pipeline at runtime, GPUs must rely on static software techniques, applied at design or compile time, to increase the instruction issue rate. This is all the more important as a controller issues the same instruction to several cores, some of which are not permitted to execute the instruction (e.g., during a branch divergence) and have to stay idle. Plus, unlike CPUs where a large hierarchy of caches automatically provides efficient access to data, GPUs mostly rely on programmers to store the data with high locality in the scarce on-chip shared memory. GPU programs also should provide synchronization among the threads in the least possible performance-damaging way, which requires a more careful design than a CPU program with less need for synchronization. Below, we briefly outline some of the prominent performance determinants in a GPU program.

\subsubsection{Occupancy}

When a kernel is launched, the GPU driver distributes each kernel block to one of the streaming multiprocessors for execution. A block/warp is considered active (aka, resident) from the time resources, such as registers and shared memory (known at compile-time), are allocated to its threads until all threads have exited the kernel. The number of active blocks/warps on an SM is limited by 
several factors imposed by the kernel launch configuration and the capabilities of the GPU. The limiting factors include the following: (a) maximum number of warps per SM that can be active simultaneously; (b) maximum number of blocks per SM that can be active simultaneously; (c) maximum number of registers per thread per SM; and (d) amount of shared memory per SM. The ratio of the number of active warps on an SM to the maximum number of active warps supported by the device is known as the theoretical occupancy. ${ }^{1}$ Maintaining high occupancy is often necessary to achieve high throughput on the GPU. The reason is that when an active warp is stalling for a long latency instruction (e.g., a memory operation), the GPU immediately replaces it with another warp ready to issue an instruction. Therefore, to mask long-latency instructions, it is desirable to have as many active warps as possible.

\subsubsection{Memory Latency}

Memory latency is the most important performance consideration in GPGPU [41]. To reduce global memory accesses, the GPU combines multiple memory accesses into one single transaction. The number of memory transactions for a certain set of memory requests and the required access pattern for achieving coalescing depend on the architecture of the GPU. In particular, it depends on the target cache (i.e., L1 or L2) that is going to hold the data and the size of the cache line (e.g., 128 bytes for L1 and 32 bytes for L2). For example, every successive 128 bytes of memory can be accessed by the threads in a warp in one coalesced 128-byte L1 transaction or four 32-byte L2 transactions. To maximize global memory throughput, it is therefore important that a program promotes memory coalescing.

\subsubsection{Branch Divergence}

When a warp reaches a flow control instruction (such as if, switch, while, and for), it successively executes every branch path that is taken by at least one of its threads. On each path, the warp deactivates the threads that do not follow that path, in order to prevent them from executing the path instructions. This can significantly impact the GPU utilization and reduce the effective instruction throughput [23]. Note that branch divergence occurs only within a warp; among warps, instructions are not executed in a lockstep fashion, thus different warps may take different paths without causing divergence. Even within a warp, divergence can completely be avoided if all the threads follow the same path; for example, when the control flow is determined by warp id, obtained as (thread id/warp size). On the other hand, the impact is more unpredictable when the control flow is determined at runtime, e.g., through

\footnotetext{
${ }^{1}$ The theoretical occupancy is the maximum occupancy that can be achieved. The actual achieved occupancy often varies at runtime and depends on various factors, such as workload distribution within and across blocks.
} 
thread-dependent data. To enhance performance, programmers should therefore try to formulate the problem so that it seldom results in thread divergence.

\subsubsection{Synchronization}

As described in Section 2.3.2, GPU programs often need to synchronize the communication between the threads using mechanisms such as barriers and atomic operations. Synchronization mechanisms create execution stalls and algorithms should be structured to minimize their use. For certain algorithms (e.g., reduction), it is possible to reduce the number of barriers (i.e., syncthreads()) by removing those that are used for intra-warp synchronization; for example, threads in a warp can use warp shuffle instructions to perform intra-warp reductions [42]. Atomic operations also impact performance, in general, because they are slower than normal memory accesses. Performance can further degrade when many threads compete to perform atomic operations on few memory locations. In such cases, competing accesses are serialized. For example, when all threads in a grid atomically access a single variable to calculate a global maximum. To mitigate serialization of accesses, local maximums can be used in order to introduce more parallelism and reduce the number of contended accesses to the same global maximum [43].

\subsubsection{Unified Virtual Memory}

In a GPU-based embedded system, the GPU is integrated into the chipset and resides on the chip alongside the multi-core CPU. The system memory that previously was only owned by the CPU is now shared between the CPU and GPU. The architecture also differs from a high-end GPU system, where the GPU has its own dedicated memory. In a unified memory architecture with a unified addressing scheme, both devices share the same virtual address space [44]. Therefore, instead of explicitly transferring the data, the CPU can pass the pointer to the input data to the GPU. Data coherency is managed either by the software at the beginning and end of the kernel launch, or by the user. Recently, cache-coherent interconnects have emerged that enforce data coherency between the CPU and GPU at the hardware level $[45,46]$. The unified memory architecture together with the cache-coherent interconnect of the heterogeneous platforms can facilitate the producer-consumer type applications, where the CPU and GPU cooperatively process a stream of data.

\subsection{Conclusion}

This chapter has provided basic background information about GPUs and has outlined some of the parameters that determine the performance of a program on a GPU. We have explained that the highly multi-threaded architecture of 
the GPU has made it suitable for efficiently processing applications that are inherently data-parallel (to fit the GPU architecture) and have large input datasets (to yield a high GPU occupancy for better hiding the long-latency operations). However, as discussed in Chapter 1, providing a large input data-set for an embedded application may result in a long latency per item, which is often undesirable. In addition, for certain applications, a large input data-set also increases the average memory latency due to cache congestion.

To address such issues, we propose several system-level techniques. In this thesis, we define a system-level technique as a software technique that, instead of dealing with optimizing the performance of a processing node individually, takes a holistic view of the requirements of the system and the relations between the processing nodes in order to make better design trade-offs. Moreover, the proposed techniques use the size of the input data-set as the common knob to balance their corresponding trade-off problems. In paper II, we control the number of image frames to maximize the system throughput, via increasing the GPU occupancy while satisfying the timing requirements. In paper III, we administer the display frame size to make a trade-off between the GPU power consumption and the quality of user experience. In paper IV, we manage the size of a batch of network packets to minimize the average packet latency while maintaining the minimum required system throughput. The technique involves maximizing the GPU occupancy and providing an efficient synchronization mechanism between the GPU threads as well as between the GPU threads and the CPU. Finally, in paper V, we control the input sizes of a number of data-dependent GPU kernels in order to increase the $\mathrm{L} 2$ cache hit rate and alleviate the memory bottleneck. Here, the challenge involves striking a good balance between a high occupancy and a good cache performance. The techniques also exploit the unified memory architecture to avoid the costly data transfer between the CPU and GPU. 



\section{Related Work}

Since the emergence of GPGPU, researchers have re-structured sequential algorithms from a wide spectrum of applications to harness the parallelism offered by GPUs and have demonstrated significant processing acceleration [47, 48] and energy savings [49] as compared to a traditional CPU environment, whether uni-core or multi-core. Apart from algorithm-level performance enhancement techniques, studies have also been carried out to facilitate several aspects of the GPGPU design, including research on modeling the power consumption of GPGPU programs [50], formal analysis techniques for reliable GPGPU programming [51], and the architecture design of GPUs [52]. In this chapter, we present the literature in each of the domains wherein this thesis has made contributions.

\subsection{GPGPU on Low-Power Embedded Systems}

Prior to our work [1], there had been a few studies looking into the potential of GPU processing in the embedded system domain. However, to the best of our knowledge, ours is the first to study the application of GPGPU in embedded systems, across various application domains, from the point of view of performance and energy efficiency. Hallmans et al. have discussed some of the requirements for moving functions from discrete units in an embedded system (like a DSP or an FPGA) into an embedded GPU in order to reduce the number of processing units. Gupta and Owens [53] have proposed compute and memory optimizations for a speech recognition application on low-end GPUs. The authors argue that the unified memory architecture in CPU-GPU SoCs results in a higher memory access contention and therefore memory optimization techniques are particularly important for these systems. The experimental results, however, do not provide information regarding the power/energy 
consumption of the proposed techniques. On the same topic, Daga et al. [54] have shown that for applications with high volume of data transfer between the CPU and GPU, a low-power embedded GPU can significantly outperform a high-end GPU, by exploiting the unified memory architecture to avoid the PCIe data transfer cost and replace it with fast memory transfers between the CPU and GPU memory partitions.

Mu et al. [55] have evaluated the potential of GPUs for high-performance computing by implementing the benchmarks from high performance embedded computing challenge benchmark suite (HPEC) on a GPU. However, the experiments of this study have been conducted on a power-hungry NVIDIA Fermi GPU, which is not a suitable representative for embedded GPUs.

Other studies in the literature have also reported performance and energy benefits of embedded GPGPU on different use cases. Leskela et al. [56] have used the OpenCL embedded profile to implement an image processing workload on a mobile device with CPU and GPU back-ends. Singhal et al. [57] have proposed techniques to optimize the performance of three image processing algorithms on an embedded GPU using OpenGL ES programming interface. Cheng and Wang [58] have also used OpenGL ES API to implement a face recognition system on a low-power mobile GPU and have demonstrated around two times the performance and energy improvements over the CPUonly implementation.

\subsection{Real-Time Processing on Embedded GPUs}

GPUs have been studied in the context of real-time systems. Researchers have investigated the opportunities posed by GPGPU in real-time systems with automotive [59] and industrial control applications [60]. In practice, however, several major challenges must be overcome before GPUs can be integrated into real-time systems, including that off-the-shelf GPU drivers often provide inadequate support for real-time scheduling in multi-tasking environments [61].

Elliott and Anderson [62] have presented two methods, based on the use of dynamic priority scheduling algorithms, to formally analyze the integration of a single GPU into a soft real-time multiprocessor environment. To remove the unguarded GPU driver from resource arbitration decisions, they have used a semaphore to avoid contentions for the GPU. The authors have also proposed GPUSync [63], which can use either static or dynamic priority GPU schedulers to manage a multi-GPU multi-core real-time system. Kato et al. [61] have proposed a scheduling framework, namely TimeGraph, that integrates the prioritization and resource reservation capabilities into the device driver. Similar to TimeGraph, the authors have also proposed a GPGPU execution model [64] to protect high-priority GPGPU tasks from competing workload. However, unlike TimeGraph, which is a device-driver solution, the proposed scheme works at the user-space level. Elliott et al. [65] have also proposed 
techniques to reduce the interference from the GPU interrupts, which can often cause complications in the analysis of real-time systems. Lee et al. [66] have advanced the previous research in [61,62], in that they consider the ability of GPUs to concurrently execute multiple applications and accordingly provide a scheduling framework by using a fine-grained GPU resource management scheme. However, the paper does not elaborate on key factors such as how the fine-grained scheduling technique is enforced on the hardware or the impact of concurrent kernels on the estimated kernel durations.

The above thread of work has presented GPU resource management techniques to address the real-time requirements in a multi-tasking GPU environment. However, in [2], we focus on a system model where the same functionality serves all streams of data arriving from multiple sources. The key challenge here is to decide which set of data items must be batched together and sent to the GPU for computation. This is different from the above works where the job creation is abstracted away. Moreover, unlike our work, these articles do not consider energy efficiency as an optimization objective.

Verner et al. $[67,68]$ have proposed algorithms for hard real-time scheduling of parallel data streams on a heterogeneous system with single- and multiGPU support. The algorithm batches multiple streams together and statically assigns them to either of the GPU(s) or CPUs. In addition to differences in the system model, our work aims to maximize energy efficiency, while these works focus on high-performance computing and try to increase the throughput of the system. Also, they consider a discrete-GPU based architecture with significant data-transfer overhead, whereas we target a GPU-integrated system, where CPU-GPU communication time is insignificant.

Research has also been conducted to compute the worst-case execution time (WCET) of applications on GPUs $[69,70,71]$. However, these techniques often face unresolved timing analysis issues in GPUs mainly caused by hardware complexities, such as shared caches, and undisclosed proprietary thread scheduling mechanisms, which make developing an effective WCET analysis framework for GPUs even more of an ambitious endeavor than it is for a multicore platform [72]. In [2], we assume that the WCET of an application is obtained through empirical tests. Recently, Santriaji et al. [73] have proposed a hardware-based resource manager for GPUs that enforces timing guarantees with minimal energy.

Recently, Lee et al. [74] have proposed a runtime scheduling framework for event-driven applications on a GPU-enabled embedded platform. The framework aims to maximize the number of real-time applications that meet their timing requirements by splitting the kernels into sub-kernels (spatial preemption) and scheduling the sub-kernel launches on the GPU according to their priorities (temporal preemption). 


\subsection{Power Management in Mobile Games}

Mobile games are among the most popular applications on handheld devices and contribute greatly to the battery drain. Several studies in the literature have investigated power management techniques for mobile games. Dietrich et al. $[75,76]$ have proposed a power management technique for embedded CPUs that scales the processor's voltage and frequency based on the current state of the game (e.g., whether the game is being loaded or played)-where the state is detected through intercepting the OS-game communication-such that the minimum desired frame rate of the state is ensured. Inspired by the DVFS-based techniques for embedded CPUs, Pathania et al. [77, 78] have proposed an integrated CPU-GPU power management scheme that exploits the DVFS capabilities in both the CPU and GPU synergistically. Recently, Hwang et al. [79] have proposed a perception-aware power optimization technique for mobile games that estimates the appropriate frame rate based on the perceptual similarity between consecutive game frames. The above techniques use the frame rate as a common knob to reduce power consumption, reasoning that the default frame rates provided by the system are often too high and user experience will not be degraded at carefully selected lower frame rates. We consider these works as orthogonal to ours [3]; we use the frame resolution as the knob, without tinkering with the frame rate.

The idea of using the resolution to save power has been briefly discussed in [80], where a GPU simulator is presented to evaluate the performance, power and image quality on mobile graphics systems. Similar to our work (published at about the same time), He et al. [81] have proposed a power management scheme using dynamic resolution scaling. Both techniques use resolution scaling as a knob to lower power consumption for closed-source games, and they both utilize quantitative analysis to account for the trade-off between power saving and user experience. However, the techniques use different approaches to achieve similar goals. First, we use a custom ROM to enforce resolution scaling at runtime, whereas their technique does not require re-building the ROM. On the other hand, the overhead of our technique is less, and it does not require intercepting the OpenGL function calls. Second, they use an ultrasonic module to accurately detect the user's distance from the device screen. The distance is then plugged into a visual acuity function, which decides the proper frame resolution in order to maintain a certain visual acuity. While it is a bright idea to integrate user's distance into the decision-making process, the technique does not capture the alterations in user preferences as the remaining battery time or the situational context of the user changes. However, we believe that these two techniques can be combined. Finally, their technique requires an additional hardware module (to measure the distance from the screen) that currently does not exist on smartphones and which consumes extra power-even though very little, as the authors claim. 
The idea of trading off quality for energy has also been studied previously. Pool et al. [82] have proposed a hardware-based approach to saving energy by reducing the precision of arithmetic operations. Recently (succeeding our work [3]), more research has been conducted in this area. Yan et al. [83] have proposed a statistical model to estimate the user satisfaction for a given application, QoS, and energy. The model is guided by the user personality, which is dynamically identified based on the device usage history. Based on the obtained user satisfaction model, the authors have further proposed a power management policy to maximize energy savings while satisfying every individual mobile user. Xie et al. [84] have proposed a perception-aware runtime technique to approximate the 3D rendering process in order to reduce the GPU power, without visible image quality loss. In their Ph.D. theses, Bischoff [85] and Devlic [86] have also investigated user-experience based optimizations for mobile systems and wireless-based video content delivery, respectively.

\subsection{Packet Processing on GPUs}

The recent interest towards software-based networking on general-purpose commodity devices has prompted researchers to exploit the highly multithreaded architecture of GPUs for parallel processing of packets. In their seminal work, Han et al. [87] have presented PacketShader, the first GPUaccelerated software router to reach multi-10 gigabits per second (precisely, 40 Gbps). PacketShader accomplishes this via significant I/O optimizations at the operating system level and efficient processing on the GPU. The GPUaccelerated applications include IPv4/6 lookup, classification, and packet encryption/authentication. Li et al. [88] have proposed GAMT, a GPU-based IPv4/6 lookup engine that can smoothly handle high update frequencies of the routing table. The proposed method encodes a multi-bit trie structure into a state-jump table efficiently represented in the GPU. Zhu et al. [89] have proposed an integrated CPU-GPU micro-architecture to optimize QoS metrics. Their work addresses two problems in GPU-based packet processing, namely the communication overhead between the CPU and GPU and the throughput vs latency trade-off. To eliminate the CPU-GPU communication overhead, the authors propose the use of a shared memory system (which is nowadays available in integrated-GPU platforms). As for the latter, they have proposed a hardware-based adaptive warp issuing mechanism that adapts to the arrival rate of network packets and maintains a balance between the throughput and worst-case packet latency. Sun et al. [90] have proposed Snap, a flexible framework that allows for packet processing tasks to be implemented as modular elements. The main feature of Snap is its ability to efficiently support complex pipelines, where packets can be split across divergent processing paths; for example, some packets can take the path through a lookup function while others pass through a pattern matching function. 
More specifically, a group of studies has focused on accelerating the packet classification [91] on GPUs. Zhou et al. [92] have proposed a two-phase algorithm. In the first phase, each thread examines a set of rules and produces a local classification result, using a binary range-tree search technique. Then in phase two, the rule with the highest priority among the local results is identified. However, the performance of this technique is limited by the size of shared memory and does not scale well with the number of rules. Varvello et al. [93] have proposed GPU-accelerated implementations of several algorithms for performing multi-layer packet classification, including linear search, tuple search, and tuple search enhanced by Bloom filters. The proposed algorithms are integrated with a high-speed packet I/O engine. Kang et al. [94] have proposed an algorithm based on a linear search framework. At the core of the framework, a metaprogramming technique compiles the rules into fragments of $\mathrm{C}$ code to avoid high-latency memory accesses required to fetch the rules.

One common pitfall in most of the previous studies is that they mainly focus on the throughput aspect and attend less to the packet latency aspect [95], perhaps due to the fact that GPUs are throughput-oriented machines and achieving a higher throughput is regarded as a more desirable goal. This, however, has an implication on the residence time of the packet inside the networking device. Addressing this issue is the main focus of our work [4].

\subsection{Addressing the Memory Bottleneck}

Memory-related stalls are considered as the main source of execution inefficiency in GPGPU programs [23]. A lot of prior efforts have been devoted to this problem, from the perspectives of hardware, software, and system-level design. Hardware-based solutions include innovations in the design of highbandwidth memory modules [96], fast on-chip interconnects [97], and efficient caches [98, 99]. Despite usually offering considerable performance improvements, these techniques are often costly and slow to deploy. Softwarebased solutions, on the other hand, seek to exploit the locality of data for more efficient data access. Memory coalescing techniques are used to exploit the spatial locality [100], while others reduce access time via keeping the recently accessed data in high-speed on-chip memory resources, such as in the registers $[34,101]$, in the user-managed scratchpad memory [102], and in the caches [103]. These software optimization techniques are flexible and fast, but they often suffer from being kernel specific. In [5], we propose a software technique that exploits the GPU L2 cache to accelerate data access. Our technique does not require prior knowledge about an application and is completely unaware of the kernel functionality.

Software techniques have been proposed to improve the cache performance in GPUs. Li et al. [104] have proposed an adaptive cache bypassing framework for GPUs to avoid cache congestion often caused by a large num- 
ber of concurrent threads competing for the same cache lines. The idea of cache bypassing is to skip placing certain data of selected threads in the cache in order to reduce cache contention, thereby increasing the effective cache capacity [105]. Li et al. [106] have also proposed a software technique to exploit the data locality between thread blocks. The technique is based on clustering the blocks with inter-block locality and reshape the default block scheduling so that all the blocks in the same cluster can be assigned to the same GPU $\mathrm{SM}$, in order to exploit the locality via SM's L1 cache. In comparison, our technique deploys kernel tiling to exploit the inter-kernel locality using the GPU L2 cache. Therefore, we believe that the two techniques can be combined to improve the performance of both L1 and L2 caches.

Tiling has also been used in other contexts to enhance the performance of GPU kernels. Rawat et al. [102] have proposed a tiling-based technique to effectively use the scratchpad memory for stencils. However, this technique can only be applied to stencils. Yun and Xiuhong [107] have developed a technique to split concurrently executing kernels at runtime to improve resource utilization. However, this technique does not deal with cache performance and, moreover, requires the kernels to be data independent. 



\section{Contributions}

\subsection{Publication List}

The content of this thesis is based on the following conference papers:

[1] A. Maghazeh, U. D. Bordoloi, P. Eles, and Z. Peng. "General purpose computing on low-power embedded GPUs: Has it come of age?" In: Proceedings of the International Conference on Embedded Computer Systems: Architectures, Modeling, and Simulation (SAMOS). Samos, Greece, July 2013, pp. 1-10.

[2] A. Maghazeh, U. D. Bordoloi, A. Horga, P. Eles, and Z. Peng. "Saving energy without defying deadlines on mobile GPU-based heterogeneous systems." In: Proceedings of the International Conference on Hardware/Software Codesign and System Synthesis (CODES+ISSS). New Delhi, India, Oct. 2014, pp. 1-10.

[3] A. Maghazeh, U. D. Bordoloi, M. Villani, P. Eles, and Z. Peng. "Perception-aware power management for mobile games via dynamic resolution scaling." In: Proceedings of the IEEE/ACM International Conference on Computer-Aided Design (ICCAD). Austin, TX, USA, Nov. 2015, pp. 613-620.

[4] A. Maghazeh, U. D. Bordoloi, U. Dastgeer, A. Andrei, P. Eles, and Z. Peng. "Latency-aware packet processing on CPU-GPU heterogeneous systems." In: Proceedings of ACM/EDAC/IEEE Design Automation Conference (DAC). Austin, TX, USA, June 2017, pp. 1-6.

[5] A. Maghazeh, S. Chattopadhyay, P. Eles, and Z. Peng. "Cache-aware kernel tiling: An approach for system-level performance optimization of GPU-based applications." In: Proceedings of Design, Automation 
and Test in Europe Conference (DATE). Florence, Italy, Mar. 2019, pp. 1-6.

In addition to the included papers, other relevant publications include

[6] E. Aragon, J. M. Jiménez, A. Maghazeh, J. Rasmusson, and U. D. Bordoloi. "Pattern matching in OpenCL: GPU vs CPU energy consumption on two mobile chipsets." In: Proceedings of the International Workshop on OpenCL (IWOCL). Bristol, UK, May 2014, 5:1-5:7.

[7] A. Maghazeh, U. D. Bordoloi, U. Dastgeer, A. Andrei, P. Eles, and Z. Peng. "Adaptive Packet Processing on CPU-GPU Heterogeneous Platforms." In: Many-core computing: hardware and software. Ed. by B. M. Al-Hashimi and G. V. Merrett. Manuscript in press.

\subsection{Publication Overview}

\section{Paper I: General Purpose Computing on Low-Power Embedded GPUs: Has It Come of Age?}

Until recently, studies on GPGPU were predominantly concerned about design and optimization of massively parallel applications on high-end GPUs. This paper focuses on GPU computing on embedded GPUs and investigates some of the opportunities and challenges in the context of low-power embedded systems. Towards this, we select five non-graphics benchmark algorithms (i.e., Rijndael algorithm, bitcount, genetic programming, convolution, and pattern matching), deployed in various embedded application, and map them to an embedded GPU. To the best of our knowledge, this is the first work to study the performance characteristics of non-graphics workloads on embedded GPUs using a real hardware platform.

Through the choice of these applications, we make the following points. First, for each application, we compare the execution time and energy consumption of the GPU implementation with the CPU implementations on single, dual, and quad cores. For the three massively parallel applications (i.e., genetic algorithm, convolution, and pattern matching), the GPU variant provides compelling performance gains over the CPU ones. For the next two memory-bound applications (i.e., Rijndael and Bitcount), the GPU provides smaller execution times as compared to the single- and double-core, but its performance is beaten by the quad-core. With respect to the energy consumption, all CPU and GPU variants exhibit similar results. ${ }^{1}$ Second, we discuss some of

\footnotetext{
${ }^{1}$ It is important to note that here we compare the performance of an embedded GPU at its early stages of development with that of a well-matured CPU core. With advances made in the past few years in the realm of embedded GPUs, today's devices provide significantly higher performance at low energy rates.
} 
the challenges in the embedded GPGPU design and demonstrate that adopting the same optimization strategies used for high-performance GPU computing may lead to poor performance on embedded GPUs. For example, because the embedded systems generally have fewer registers in their streaming multiprocessors, the same kernel designed and optimized for a high-end GPU if implemented on an embedded GPU could potentially result in register spilling and more memory accesses, hence lower performance. ${ }^{1}$ Finally, based on the promising results on a yet premature device, we envision that a new wave of computationally heavy applications that are conventionally known to be prohibitively expensive to run on embedded systems may eventually become feasible as the architecture of embedded GPUs evolves.

\section{Paper II: Saving Energy Without Defying Deadlines on Mobile GPU-Based Heterogeneous Systems}

In embedded systems, energy is at a premium. Therefore, energy-efficient computing is of great significance. The high throughput and energy efficiency of GPUs seem to well meet the high computational demands of these systems. However, many embedded applications have real-time requirements, which do not readily adhere to the throughput-oriented design of GPUs. In this paper, we propose a scheduling scheme for a real-time system based on the cooperative use of a GPU and multiple CPU cores in a heterogeneous platform.

The input to the system is a set of streams. Each stream generates a sequence of items at regular intervals-unique to each stream. items must be processed either on a CPU core or on the GPU before a certain deadline. Moreover, items are processed by the same function, even though each is associated with a different input data. Our goal is to provide a schedule to minimize the overall energy consumption of processing the items while satisfying their deadlines. One example of such a system model can be found in self-driving cars where multiple cameras stream data frames for sensing the surroundings. The frame rates of cameras may vary, for example, due to the different device characteristics or the criticality of the camera position relative to the car movement.

Using GPUs for real-time applications poses several challenges, at the heart of which lies the trade-off between throughput (subsequently energy efficiency) versus latency. GPUs are inherently more energy efficient than latencyoriented CPU cores, but they require a minimum amount of workload in order to deliver high throughput-per-watt; otherwise, it is more energy efficient to assign the task to the CPU cores. Moreover, due to the inherent characteristics of some applications and the fact that GPUs are steadily becoming more re-

\footnotetext{
${ }^{1}$ We note that some of the challenges discussed in the paper have now been resolved via producing more resourceful embedded GPUs or by unifying the architecture of streaming multiprocessors in embedded and high-end GPUs, for example, in NVIDIA Tegra. However, even then and due to the characteristics and requirements of the embedded systems (like power related restrictions), designing kernels for embedded GPUs may require special treatments.
} 
sourceful, it is reasonable to assume that the corresponding workload of a single item is not sufficiently large to sustain a high GPU utilization rate. Therefore, to meet the minimum workload requirement, multiple items should be collected and processed together as a single larger job on the GPU. However, determining the job size (i.e., the number of items in a job) is not a trivial problem, for two primary reasons: First, increasing the job size results in a larger waiting and processing time for the constituent items. Consequently, some of the items may miss their deadlines or be forced to run on the less energy efficient CPU cores in order to meet their deadlines. Second, a combination of a large job followed by a small one may consume more energy than two mediumsize jobs. These factors indicate the need for an efficient, yet thorough, design space exploration technique.

We propose an iterative heuristic to solve the scheduling problem. In each step, the heuristic finds the most energy-efficient schedule for the items that arrive during a given time interval, called the simulation interval, using a local search algorithm. Initially, the simulation interval is set to one hyperperiod of the system. ${ }^{1}$ The local search algorithm then finds the energy-optimized static schedule for the given interval by transforming the problem into a problem of finding the shortest path in a directed acyclic graph. Once the schedule is found, the heuristic increases the simulation interval by one hyperperiod and performs the local search again, aiming to discover a better schedule. This procedure repeats iteratively until the heuristic terminates at any step where (a) no feasible schedule can be found, or (b) the best-found local schedule cannot improve the best-found global result, or (c) a predefined timeout is reached. The obtained schedule determines the exact processing time of the items as well as their mappings to either of the GPU or the CPU cores. We validate the effectiveness of our technique by performing several sets of experiments on a development board and demonstrate major improvements in the energy consumption of the board in various configurations. We also report superior energy performance for the schedules proposed by our heuristic when compared to the schedules proposed by an ILP solver within a one-hour time limit.

\section{Paper III: Perception-Aware Power Management for Mobile Games via Dynamic Resolution Scaling}

In the everlasting race towards visually appealing mobile devices, displays have experienced a significant increase in size and resolution. As a result, the number of frame pixels rendered by the GPU have increased dramatically, leading to a higher power consumption by the GPU and a shorter battery discharge time. Meanwhile, the majority of users barely notice any image quality degradation over marginal reductions of the display resolution, or they may be will-

\footnotetext{
${ }^{1}$ The actual size of the simulation interval may slightly be larger than one hyperperiod due to hyperperiod spilling.
} 
ing to trade image quality (by accepting lower resolutions) for a longer battery time, all the more so when currently it is not possible to charge the device. In this paper, we propose a power management scheme for mobile games by dynamically changing the display resolution while the game is being played. Our technique uses a decision theory model to combine the qualitative metrics (i.e., user preferences) with the quantitative metrics (i.e., the remaining battery time) in order to make the best decision.

First, we show that resolution scaling provides a significant potential for saving power and battery time. To this end, we select 12 popular Android games and play each game at six different resolutions. We provide the knob that allows us to dynamically change the resolution for closed source games by slightly modifying the Android framework source code. At runtime, upon system's request to lower the resolution, the knob enforces the new resolution by reducing the size of the buffer into which the frame pixels are rendered.

Next, we propose the power management scheme that exploits the knob to provide the best user experience for the longest battery time. The manager begins with the start of a game session and runs until the session terminates. During this time, upon occurrences of certain events (e.g., when the battery level drops to certain levels, or when there is a sudden change in the system power), an algorithm is invoked to select the most appropriate resolution from a set of candidate resolutions, based on the current conditions. The manager then uses the knob to switch to the new resolution.

The algorithm that selects the next resolution uses a mathematical model to determine which candidate resolution provides the best trade-off between quality and power, considering a given state of the system (i.e., the remaining battery charge and the current system power) and the situational context (e.g., whether it is possible to charge the device). The model quantifies the suitability of individual candidate resolutions by calculating an expected loss value for each. The value encapsulates two factors: (a) the probability with which the user would choose a certain candidate under normal conditions, and (b) a tunable parameter that allows the user to alter their normal preferences in special circumstances, for example, while traveling and not being able to charge the device. Upon invocation, the algorithm selects the candidate with the least expected loss value.

To obtain the probabilities of different user choices, we use a classification method based on the multinomial logistic regression model. The model statistically relates the observed user choices to the amount of the leftover time corresponding to each candidate resolution. We estimate the parameters of the classifier by conducting an offline user survey, where users give their preferences after having been shown a demo of a game played at the candidate resolutions on the target device. Once the model is trained, we provide the estimated leftover times, for the current system state, as the input to the model and obtain the probability associated with each candidate resolution. 
We estimate the leftover time for each resolution by using a function of the remaining battery time and the estimated system power at a given resolution. The former can easily be acquired at any moment through the Android APIs. As for the latter, we develop a power model to obtain the overall system power by estimating the power of every major system component (CPU, GPU, display, etc.). We build on the model that Android uses to determine the battery usage statistics per application. Android does not provide the power rates for the GPU and memory unit. Therefore, we build a GPU and a memory power model. The GPU model estimates the power using the current utilization of the GPU and its operating frequency. As for the memory unit, however, Android does not provide the utilization rate. Therefore, we use the GPU utilization to estimate the memory power, based on the empirical observation that there is a strong correlation between the GPU and memory utilizations during the game. For each of the GPU and memory models, we obtain an accuracy of $97.4 \%$ and $98.4 \%$, respectively.

We evaluate our power management technique by conducting an experiment that shows how the system switches from one resolution to another as a new event arises. We also compare the battery depletion time when our approach is used with the ones when the resolution is fixed and demonstrate the effectiveness of the technique in extending the battery life.

\section{Paper IV: Latency-Aware Packet Processing on CPU-GPU Het- erogeneous Systems}

Recently, there has been an interest in software implementation of packet processing applications on general-purpose processors. GPUs appear to suit this purpose, given that as a router processes millions of packets per second, it creates a huge amount of data parallelism. In this paper, we propose a softwarebased packet processing approach on low power heterogeneous platforms. We exploit the computing power of GPUs to fulfill the high throughput demand of packet processing applications while addressing their timing requirements.

The main challenge of using GPUs for packet processing is to reconcile the conflicting goals of achieving a high throughput while keeping a low latency per packet. To achieve a high throughput, large batches of packets must be gathered before being dispatched-as the workload per single packet is far below that required to efficiently utilize the GPU. But large batches entail a large latency. Small batches, by contrast, provide a low latency but have a low throughput. Instead of maximizing the throughput by constantly making large batches, we propose to dynamically change the batch size according to the traffic rate. At runtime, the system regularly monitors the input rate and selects the minimum batch size (from an offline table of batch sizes and their corresponding throughputs) that provides the smallest throughput that is higher than the input rate. Thereafter, until another batch size is selected, the GPU processes the packets in batches of the newly selected size. 
The switching between batch sizes must be swift, however, to avoid a major throughput loss. An intuitive way to enforce a new batch size is to invoke a separate GPU kernel launch for every batch and set the grid size accordingly. This solution is crude. The first reason is the kernel launch overhead. Although for typical GPGPU applications with execution times in the order of milliseconds (or higher) the launch overhead is negligible, for packet processing applications with latency requirements in the order of microseconds this overhead cannot be ignored. The second reason is the workload imbalance across blocks, which happens when blocks that finish early cannot be replaced by blocks of a new kernel until all currently running blocks complete. Both reasons have a more detrimental impact on throughput when the workload (i.e., batch size) is small. Thus, we propose to use a persistent kernel to mitigate these issues.

The main characteristic of a persistent kernel is that it is launched only once and runs continuously (in an infinite loop) until terminated by the host. The kernel is composed of several blocks that are resident at all time during the lifetime of the kernel, as opposed to a conventional kernel where blocks are constantly replaced. Once a block processes a batch, it busy-waits until the next batch becomes available. A persistent kernel does not suffer from the performance issues of frequent launching. First, the kernel launch overhead is eliminated. Second, unlike the conventional approach, the blocks can work on different batches concurrently and hence avoid the workload imbalance.

We also propose a software architecture that exploits the unified memory architecture in GPU-based heterogeneous systems to process the packets in cooperation with the CPU. Using this feature, the GPU can directly access the memory to read the packet headers that are stored in a receive buffer earlier by the host. Likewise, the CPU can directly access the results from the GPU that are stored in a transmit buffer. We also use the unified memory to coordinate the tasks between the CPU and GPU, for example by seamlessly communicating the batch sizes while the kernel is running. Moreover, several threads on a multi-core CPU perform the tasks of (a) initializing the system, (b) reading back the results produced by the GPU, and (c) monitoring the traffic rate and deciding the appropriate batch sizes.

As a case study, we use a packet classification algorithm and implement it on a state-of-the-art development board according to our proposed technique. We then conduct experiments, using realistic traffic patterns, and show that the adaptive technique can provide a lower average packet latency as compared to the conventional fixed-size batch processing. While the technical details are specific to packet processing, the presented software architecture and the proposed techniques to handle the latency-throughput trade-off are generally applicable to any application with similar characteristics. 


\section{Paper V: Cache-Aware Kernel Tiling: An Approach for System- Level Performance Optimization of GPU-Based Applications}

Memory latency is a major performance bottleneck in GPU computing applications. Common software-based memory optimization techniques are based on the idea of holding the data in fast on-chip memory types (e.g., registers, scratchpad, and cache memory) after it is initially loaded from the memory. However, the overhead of the initial access can be prohibitive. Moreover, we observe that many real-life applications consist of multiple kernels with data dependencies between them, meaning that the output of one kernel is passed as the input to its successor(s). Therefore, we propose an approach that exploits the GPU L2 cache to accelerate data passing among adjacent kernels in a GPU-based application graph. The idea is that instead of processing the entire input by each kernel in one kernel launch, we split the input and process each fragment completely by all kernels before starting with the next fragment. This way, ideally, the initial data accesses are carried out via the GPU L2 cache. Our proposed technique requires minimal modification of the source code and is completely oblivious to the particularities of the kernels.

To begin with, we identify three conditions that a kernel must satisfy in order to benefit from the improved cache performance that is provided by the tiling technique. First, there should be a relatively large gap between the cache hit rate when the kernel processes the entire input (without tiling) and the cache hit rate when the kernel runs at the minimum grid size (i.e., one block per kernel). Second, the overall system performance must be limited by memory accesses. And third, data dependencies of the kernel blocks must not depend on the input data. This is required so that, offline, we can determine the dependencies, which are required to preserve the functional correctness of the application. Using the example of a kernel that satisfies the three conditions, we show the performance superiority of a small kernel with a high cache hit rate over a large kernel with a low hit rate. We propose a tool that builds on this observation to maximize the performance of the tiling-eligible kernels in an application graph and minimize the overall duration of the application.

Our tool is composed of two main modules: a block analyzer and a block scheduler. The block analyzer gets as input the application source code and a sample set of application inputs with a given size. It then produces the data dependencies among all kernel blocks and a list of all memory lines that are accessed by each block. This information is passed to the block scheduler which then determines the size of each sub-kernel and the blocks assigned to them, as well as the order in which the sub-kernels are executed on the GPU.

The block analyzer constructs a dependency graph that captures the data dependencies between kernel blocks. Towards this, it analyzes a memory trace which contains the memory addresses accessed by the individual threads of each block. To record the memory trace, we use an instrumentation tool which allows us to inject code before all global memory instructions and obtain the 
target memory address. Next, we process the trace and obtain all dependencies. Using the same memory trace, we also collect a list of all memory lines that each block accesses during a full execution of the kernel. This is used by the scheduler to calculate the memory footprint of the sub-kernels in order to avoid creating oversized sub-kernels with large memory footprints that result in more cache conflicts.

The scheduler module uses an iterative heuristic to decide which kernels to tile and how to tile them. In the process, it also uses a set of user-provided information to account for the performance characteristics of each kernel on the specific platform. At each iteration, the heuristic first partitions the nodes (i.e., kernels) into disjoint clusters. To decide the nodes that are put in the same cluster, the heuristic considers each kernel's potential for performance improvement (using the information provided by the user). For example, if a kernel's duration can significantly be reduced by tiling, that kernel and its predecessor kernel (that produces the input) are clustered together. Then, the heuristic tiles the nodes in each cluster and estimates the overall execution time of the cluster. The heuristic accepts any new cluster that reduces the overall duration of its member nodes and rejects it otherwise. It then attempts to expand the clusters in the following iterations by merging the adjacent clusters. This procedure repeats until there is no room left for further improvement. Upon termination, the heuristic returns the schedule corresponding to the tiling of the clusters in the best-found partition.

We apply our tool to a full-fledged image processing application with over a thousand kernels and evaluate its effectiveness under various GPU/memory frequency configurations. On average, we achieve a performance improvement of $30 \%$.

\subsection{Discussion}

In the previous section, we discussed the contributions of each paper individually. Here, we discuss some of the applications of the proposed techniques in the context of today's complex systems. In particular, we provide a few scenarios where exploiting the synergy between two or more of the techniques can improve the efficiency of the system while fulfilling its requirements.

As technology evolves and GPUs become more capable, a single device can service more tasks simultaneously. Therefore, instead of having several processors to handle different tasks separately, those tasks can share the same GPU. This can prevent over-designing and save cost, chip space, and power. However, shifting from a distributed application-specific configuration to a centralized shared configuration inflicts a heavy burden on the system-level software design. In the following, we present three scenarios where, in each scenario, one of the proposed techniques or a combination of them is used to efficiently handle a multi-aspect problem. 
1. Managing screen resolution in a multi-display setup. For this scenario, consider a vehicle with four infotainment displays that use the GPU for rendering the graphics content. Suppose a single GPU is at most capable of rendering one stream at $4 \mathrm{k}(3840 \times 2160)$ resolution. In this case, one simple but extravagant design choice is to use four GPUs-one for each display. Another alternative is to fix the default resolution of every display at $1080 p(1920 \times 1080)$ and render all applications at this size with a single GPU. This solution is also not desirable because, for example, if only one display is turned on, the viewer should have been able to enjoy the highest resolution. Instead, we can use an adaptive approach (proposed in paper III) to dynamically adjust the resolutions according to the number of active displays. For example, when a second display is turned on, the resolution of each active display is set to half of $4 \mathrm{k}$ (e.g., $2715 \times 1527)$ and when either is turned off, the resolution of the other display jumps up to $4 \mathrm{k}$. In a more intricate scenario, applications can be associated with different desirability rates at different resolutions. Then at runtime, an online manager solves an optimization problem to find the appropriate resolution for each display.

Additionally, the same GPU can be used even by non-graphics applications. For example, it can be utilized to process camera streams for a computer vision application. In this case, as soon as the computer vision application becomes active (e.g., when the car is put in the autonomous driving mode) the manager readjusts the screen resolutions in order to accommodate the higher priority task on the GPU.

2. Cache-aware scheduling of real-time applications. This scenario illustrates the synergy between batch processing in real-time applications (proposed in paper II), as a means to improve the GPU throughput, and cache-aware kernel scheduling (proposed in paper $\mathrm{V}$ ), to reduce the memory access time. As an example, consider a computer vision system where several camera streams are fed to a GPU and every frame has to go through a GPU-based application graph (e.g., optical flow). As processing a single frame on a powerful GPU may lead to a low GPU utilization, multiple frames are batched and processed together, provided that the timing constraint of each frame is satisfied. In addition to the batch processing technique, it may be possible to further improve the performance by utilizing the GPU L2 cache as a fast means to communicate the intermediate data between consecutive kernels. While increasing the batch size improves the utilization, it may, however, reduce the cache performance due to a larger memory footprint. This contradiction in objectives can be converted into an optimization problem, whose solving requires to find the appropriate batch sizes that can provide a good trade-off between a high GPU utilization and a high cache hit rate. 
3. Assured service provisioning for latency-sensitive applications. The lack of support for task preemption in GPUs can create a challenge when multiple applications with different priorities share the same GPU. To underscore this matter, consider a GPU-based packet processor where multiple packet streams with different classes of service must be processed by the same GPU. In the normal kernel execution mode, processing a batch of packets with high priority does only start after the currently running kernel is completed. This waiting time introduces an additional latency and jitter to the latency-sensitive packets. One way to circumvent this problem is to permanently allocate a part of the GPU resources to the high-priority packets. This can be achieved by using a persistent kernel such that the threads of the designated blocks only process the packets from the high-priority buffer-and busy-wait in case the buffer is empty. Although this technique may affect the GPU utilization due to constantly reserving part of the resources, it precludes latency variation due to low-priority interference and provides a more reliable service for high-priority packets.

An alternative to this approach is to use the stream priority feature in CUDA. Prioritizing the packets in this way, however, does not eradicate the low-priority interference. The reason is that even though the high-priority blocks will be scheduled to run first, they still may not preempt the low-priority blocks and need to wait until the currently running blocks finish their tasks, thereby once again increasing the latency and jitter of the high-priority packets. 



\section{Conclusions and Future Work}

In today's embedded design, system-level methodologies are more crucial than ever. As the need for processing power in complex systems, such as autonomous vehicles, continues to rise, designers face increasing challenges, including tight constraints on power and cost. Meanwhile, with the latest advances in the GPU technology, a single embedded GPU is theoretically able to satisfy the computation demands of several applications combined. This creates the opportunity to replace multiple devices on the chip with a single GPU, and by doing so reduce cost and power. However, realizing this opportunity requires careful system-level software design, as GPUs are power-hungry devices and if not utilized efficiently, they can create debilitating power-related issues. The objective of this thesis has been to contribute to this cause by proposing several system-level techniques to improve the performance of GPU-based embedded platforms. In this chapter, we recapitulate the main contributions of the thesis and suggest directions for future work.

\subsection{Conclusions}

The central question of this thesis has been how to optimize the performance of a GPU-based embedded system under one or a set of applications. In response, the thesis has presented several system-level software techniques to improve the performance of the system, under graphics and non-graphics applications. This system-level approach is unlike the one in the previous body of research, which, for the most part, has focused on the application-level techniques to optimize the performance of a specific application on the GPU. The difference in perspective is mainly driven by the fact that embedded systems are restricted by several constraints (i.e., power, throughput, and time-related constraints) and often the design must be optimized with respect to more than one con- 
straint. Therefore, in addition to low-level application-specific optimizations, a high-level view of the system is required to capture the various requirements. For example, a design optimized to only maximize the throughput may not be able to fulfill the timing constraints. Another factor that distinguishes the embedded system design from high-performance computing is the application type. An embedded system often deals with streaming applications, where processing a single item on the GPU results in low device utilization. This contrasts with designing for high-performance computing, where the input is considered to be arbitrarily large. Apart from such challenges, the oblivion of a system-level design towards application specificities enables it to be generally applicable to a broader range of applications with similar characteristics.

After analyzing some of the challenges and opportunities of embedded GPU computing in paper I, we have proposed four techniques to improve different performance aspects of the system under various deployment scenarios. In the broadest sense, the common means to accomplish the objective in each technique has been to manage the workload on the GPU by controlling the input size. In paper II and paper IV, we have used this factor to decide the number of items that must be batched and dispatched together to the GPU in order to achieve a high throughput without sacrificing the timing requirements. In paper III, we have proposed a method for graphics applications (in particular, for mobile games) to decrease the GPU workload by reducing the number of pixels in a frame. In paper $\mathrm{V}$, we have developed a tool to split a kernel with a large input into multiple smaller sub-kernels in order to increase the GPU L2 cache hit rate and thereby diminish the memory bottleneck. We have also discussed a few hypothetical scenarios where some of the proposed techniques can be applied in tandem to serve the needs of multiple applications simultaneously. We note that all our studies have been supported by empirical results obtained from real-life experiments on hardware platforms.

In the upcoming era of autonomous vehicles and similar technologies, GPUs can play a central role as the data-processing hub in the system. But, in this regard, they are strongly rivaled by other accelerators including FPGAs, DSPs, and application- and domain-specific co-processors. For the particular applications at hand, these processors often provide outstanding performance at low power rates. In comparison, the GPU's primary strengths lie in its generality and ease of programming. We believe that system-level techniques that facilitate sharing and utilizing the GPU more efficiently enable the GPU to deliver a performance on a par with other specialized accelerators, while also allowing for smaller and cheaper platforms as well as faster time-to-market. This thesis has been an early attempt to address some of the issues towards this goal. However, there are still numerous challenges that remain to be solved. In the next section, we discuss a few open problems. 


\subsection{Future Work}

In Section 4.3, we have discussed a few scenarios where variations of our proposed techniques could be used in a GPU-based complex system. This section outlines other directions for future research based on the thesis work.

Thermal issues are among the main challenges that designers must overcome when deploying GPUs in embedded systems. The problem stems from the fact that, despite being energy-efficient computing devices, GPUs usually consume high amounts of power. This can rapidly increase the temperature of the chip and the underlying circuit board and hence create heat-related issues, such as performance throttling. In the thesis, we have not addressed the thermal issues directly. However, our proposed techniques may have indirect impacts on the thermal performance of the GPU; for example, by utilizing the GPU more effectively via batching the items (proposed in paper II), by reducing the GPU power consumption via resolution scaling (proposed in paper III), or by reducing the memory power consumption (proposed in paper V). However, more research is required in this area to determine the effectiveness of a high-level design in finely managing the thermal behavior of the GPU.

We now discuss a possible progression for each of the techniques. In paper II, we have provided a scheduling mechanism to improve the GPU throughput by batching multiple items from different input streams. Our heuristic has been based on the assumption that all items have similar relative deadlines. However, it would also be interesting to design a heuristic for a system model where streams have different relative deadlines.

In paper III, we have used the frame resolution in mobile games as a knob to reduce the GPU workload, and hence reduce its power consumption. As discussed in Chapter 2, another line of research also strives to reduce the system power by using an orthogonal scheme, that is, through scaling down the frame rate of the game via dynamic frequency scaling techniques. As a future study, it would be interesting to develop a perception-aware power management scheme that exploits both resolution and frame rate scaling in a harmonious manner to optimize the power consumption without hampering the user experience. The next step would be then to investigate whether it is possible to integrate a mechanism into such a system to empower it to detect the dynamics of the game (i.e., game speed). ${ }^{1}$ This feature would allow the system to provide a better user experience by reducing the power at any moment through the knob that has a less visual impact on the current state of the game. For example, the system may choose to maintain a high frame rate at high game speeds, in order to avoid a choppy user experience and compensate by lowering the resolution (and vice versa). The technique may also be extended to include other graphics applications beyond mobile games.

\footnotetext{
${ }^{1}$ Recently, a technique that can identify the appropriate frame rate based on the frame content has been proposed in [79].
} 
The proposed technique in Paper IV deals with implementing one packetprocessing application (i.e. packet classification) using a persistent kernel on the GPU. In further research, it may be worthwhile to implement other applications that manipulate the packet inside the router, using the same approach. The next natural step is then to map on the GPU a part or the entirety of the packet flow diagram, which consists of several processing nodes performing various functions, e.g., encryption/decryption or pattern-matching algorithms. This can be achieved by implementing each application as a distinct persistent kernel residing alongside other persistent kernels on the GPU. In this way, the flow can be seen as a pipeline and each application as a stage of the pipeline. In addition to the advantages of the persistent kernel and a lower packet latency, this approach may also increase the throughput due to pipelining. However, further work is required to investigate if the achieved performance gain can outweigh the detrimental effects caused by the imbalance among pipeline stages and the possible reduction of GPU utilization due to persistent kernels.

In paper $\mathrm{V}$, we have proposed a static scheme to improve the performance of GPU-based application graphs by splitting certain memory-bound kernels into smaller sub-kernels. However, a considerable number of kernels are compute-bound. As shown in previous studies, interleaving the memory- and compute-bound kernels can significantly increase the GPU utilization, provided that the kernels are sufficiently small so that both can run concurrently on the GPU. Therefore, a further study needs to examine whether it is possible to develop a tiling scheme that, in addition to improving the cache performance, considers the performance benefits of a mixed workload. Despite the potential for significant performance improvement, this approach entails several challenges, among which the chief one is to accurately predict the duration of a sub-kernel when its execution is partially or entirely overlapped with other sub-kernels. This problem is hard because even a small error in prediction could lead to a total misalignment of the predicted execution timeline with respect to the timeline produced at runtime. Another application that can potentially benefit from kernel tiling is the case of neural networks. Neural networks involve running a tremendous amount of matrix operations on the GPU. As discussed in the thesis, some matrix operations lend themselves well to tiling. Therefore, we recommend that a further study is undertaken to investigate whether tiling may be used to accelerate the execution of neural networks on GPUs. Finally, we suggest that similar cache-aware techniques be explored in the context of upcoming heterogeneous platforms, where processors can snoop into one another's caches or share a higher-level cache. 


\section{Bibliography}

[1] A. Maghazeh, U. D. Bordoloi, P. Eles, and Z. Peng. "General purpose computing on low-power embedded GPUs: Has it come of age?" In: Proceedings of the International Conference on Embedded Computer Systems: Architectures, Modeling, and Simulation (SAMOS). Samos, Greece, July 2013, pp. 1-10.

[2] A. Maghazeh, U. D. Bordoloi, A. Horga, P. Eles, and Z. Peng. "Saving energy without defying deadlines on mobile GPU-based heterogeneous systems." In: Proceedings of the International Conference on Hardware/Software Codesign and System Synthesis (CODES+ISSS). New Delhi, India, Oct. 2014, pp. 1-10.

[3] A. Maghazeh, U. D. Bordoloi, M. Villani, P. Eles, and Z. Peng. "Perception-aware power management for mobile games via dynamic resolution scaling." In: Proceedings of the IEEE/ACM International Conference on Computer-Aided Design (ICCAD). Austin, TX, USA, Nov. 2015, pp. 613-620.

[4] A. Maghazeh, U. D. Bordoloi, U. Dastgeer, A. Andrei, P. Eles, and Z. Peng. "Latency-aware packet processing on CPU-GPU heterogeneous systems." In: Proceedings of ACM/EDAC/IEEE Design Automation Conference (DAC). Austin, TX, USA, June 2017, pp. 1-6.

[5] A. Maghazeh, S. Chattopadhyay, P. Eles, and Z. Peng. "Cache-aware kernel tiling: An approach for system-level performance optimization of GPU-based applications." In: Proceedings of Design, Automation and Test in Europe Conference (DATE). Florence, Italy, Mar. 2019, pp. 1-6.

[6] E. Aragon, J. M. Jiménez, A. Maghazeh, J. Rasmusson, and U. D. Bordoloi. "Pattern matching in OpenCL: GPU vs CPU energy con- 
sumption on two mobile chipsets." In: Proceedings of the International Workshop on OpenCL (IWOCL). Bristol, UK, May 2014, 5:1-5:7.

[7] A. Maghazeh, U. D. Bordoloi, U. Dastgeer, A. Andrei, P. Eles, and Z. Peng. "Adaptive Packet Processing on CPU-GPU Heterogeneous Platforms." In: Many-core computing: hardware and software. Ed. by B. M. Al-Hashimi and G. V. Merrett. Manuscript in press.

[8] CUDA Toolkit Documentation. Version 10.0. https://docs.nvidia. com/cuda.

[9] The OpenCL Specification. Version 2.2. https://www . khronos .org/ registry/OpenCL.

[10] I. K. Park, N. Singhal, M. H. Lee, S. Cho, and C. Kim. "Design and performance evaluation of image processing algorithms on GPUs." In: IEEE Transactions on Parallel and Distributed Systems 22.1 (Jan. 2011), pp. 91-104.

[11] S. N. Sinha, J.-M. Frahm, M. Pollefeys, and Y. Genc. "Feature tracking and matching in video using programmable graphics hardware." In: Machine Vision and Applications 22.1 (Jan. 2011), pp. 207-217.

[12] J. Agulleiro, F. Vázquez, E. Garzón, and J. Fernández. "Hybrid computing: CPU+GPU co-processing and its application to tomographic reconstruction." In: Ultramicroscopy 115 (2012), pp. 109-114.

[13] Y. Wang, H. Du, M. Xia, L. Ren, M. Xu, T. Xie, G. Gong, N. Xu, H. Yang, and Y. He. "A hybrid CPU-GPU accelerated framework for fast mapping of high-resolution human brain connectome." In: PLOS ONE 8 (May 2013), pp. 1-14.

[14] S. Solomon, R. K. Thulasiram, and P. Thulasiraman. "Option pricing on the GPU." In: IEEE International Conference on High Performance Computing and Communications (HPCC). Sept. 2010, pp. 289-296.

[15] Y.-L. Zhu, H. Liu, Z.-W. Li, H.-J. Qian, G. Milano, and Z.-Y. Lu. "GALAMOST: GPU-accelerated large-scale molecular simulation toolkit." In: Journal of Computational Chemistry 34.25 (2013), pp. 2197-2211.

[16] E. Alerstam, T. Svensson, and S. Andersson-Engels. "Parallel computing with graphics processing units for high-speed Monte Carlo simulation of photon migration." In: Journal of Biomedical Optics 13 (Nov. 2008), p. 060504.

[17] K. J. O'Dwyer and D. Malone. "Bitcoin mining and its energy footprint." In: IET Irish Signals Systems Conference and China-Ireland International Conference on Information and Communications Technologies (ISSC/CIICT). June 2014, pp. 280-285. 
[18] A. Krizhevsky, I. Sutskever, and G. E. Hinton. "ImageNet classification with deep convolutional neural networks." In: Proceedings of the International Conference on Neural Information Processing Systems. Vol. 1. 2012, pp. 1097-1105.

[19] D. Amodei et al. "Deep speech 2: end-to-end speech recognition in English and Mandarin." In: Proceedings of the on Machine Learning. Vol. 48. 2016, pp. 173-182.

[20] G. E. Moore. "Cramming more components onto integrated circuits." In: Proceedings of the IEEE 86.1 (Jan. 1998), pp. 82-85.

[21] R. H. Dennard, F. H. Gaensslen, V. L. Rideout, E. Bassous, and A. R. LeBlanc. "Design of ion-implanted MOSFET's with very small physical dimensions." In: IEEE Journal of Solid-State Circuits 9.5 (Oct. 1974), pp. 256-268.

[22] P. B. Hansen, E. W. Dijkstra, and C. A. R. Hoare. The Origins of Concurrent Programming: From Semaphores to Remote Procedure Calls. Springer-Verlag, 2002.

[23] CUDA C Programming Guide. https : / / docs . nvidia . com / cuda / cuda-c-programming-guide/, retrieved in Oct. 2018. NVIDIA.

[24] D. A. Patterson and J. L. Hennessy. Computer Organization and Design, Revised Fourth Edition, Fourth Edition: The Hardware/Software Interface. 4th. Morgan Kaufmann Publishers Inc., 2011.

[25] D. B. Kirk and W.-m. W. Hwu. Programming Massively Parallel Processors, Third Edition: A Hands-on Approach. 3rd. Morgan Kaufmann Publishers Inc., 2016.

[26] S. Collange, D. Defour, and A. Tisserand. "Power consumption of GPUs from a software perspective." In: Proceedings of the International Conference on Computational Science. May 2009, pp. 914-923.

[27] D. P. Rodgers. "Improvements in multiprocessor system design." In: SIGARCH Computer Architecture Nerws 13.3 (June 1985), pp. 225-231.

[28] S. Mittal and J. S. Vetter. "A survey of CPU-GPU heterogeneous computing techniques." In: ACM Computing Surveys (CSUR) 47.4 (July 2015), 69:1-69:35.

[29] E. Lindholm, M. J. Kilgard, and H. Moreton. "A user-programmable vertex engine." In: Proceedings of the 28th Annual Conference on Computer Graphics and Interactive Techniques. SIGGRAPH '01. 2001, pp. 149-158.

[30] S. Morein, L. Lefebvre, A. Gruber, and A. Skende. "Graphics processing architecture employing a unified shader." Patent US6897871B1. Nov. 2003. 
[31] E. Lindholm, J. Nickolls, S. Oberman, and J. Montrym. "NVIDIA Tesla: A unified graphics and computing architecture." In: IEEE Micro 28.2 (Mar. 2008), pp. 39-55.

[32] NVIDIA Tesla V1OO GPU Architecture. https : / / images . nvidia . com / content / volta - architecture / pdf / volta - architecture whitepaper.pdf, retrieved in Oct. 2018. NVIDIA.

[33] A. Cano. "A survey on graphic processing unit computing for largescale data mining." In: Wiley Interdisciplinary Reviews: Data Mining and Knowledge Discovery 8.1 (Nov. 2017), e1232.

[34] A. Li, S. L. Song, A. Kumar, E. Z.Zhang, D. Chavarría-Miranda, and H. Corporaal. "Critical points based register-concurrency autotuning for GPUs.” In: Design, Automation and Test in Europe Conference (DATE). Mar. 2016, pp. 1273-1278.

[35] H. G. Cragon. Memory Systems and Pipelined Processors. USA: Jones and Bartlett Publishers, Inc., 1996. ISBN: 0-86720-474-5.

[36] OpenMP Application Programming Interface. Version 4.5. https : / / www. openmp.org.

[37] The OpenACC Application Programming Interface. Version 2.6. https://www.openacc-standard.org.

[38] J. Enmyren and C. W. Kessler. "SkePU: A multi-backend skeleton programming library for multi-GPU systems." In: Proceedings of the International Workshop on High-level Parallel Programming and Applications. Sept. 2010, pp. 5-14.

[39] NVIDIA's Next Generation CUDA Compute Architecture: Kepler GK11O. https : / / www . nvidia . com / content / pdf / kepler / nvidiakepler - gk110 - architecture - whitepaper . pdf, retrieved in Oct. 2018. NVIDIA.

[40] M. Harris and K. Perelygin. Cooperative Groups: Flexible CUDA Thread Programming. https : / / devblogs . nvidia . com / cooperative-groups/, retrieved in Oct. 2018. NVIDIA.

[41] CUDA C Best Practices Guide. http://docs .nvidia.com/cuda/cudac-best-practices-guide, retrieved in Oct. 2018. NVIDIA.

[42] S. Matwankar. CUDA 7.5: Pinpoint Performance Problems with Instruction-Level Profiling. https : / / devblogs . nvidia . com / cuda7 - 5 - pinpoint - performance - problems - instruction - level profiling/, retrieved in Oct. 2018. NVIDIA.

[43] J. Balfour. CUDA Threads and Atomics. https : / /mc . stanford.edu / cgi-bin/images/3/34/Darve_cme343_cuda_3.pdf, retrieved in Oct. 2018. NVIDIA. 
[44] N. Wilt. CUDA Handbook: A Comprehensive Guide to GPU Programming. 1st. Addison-Wesley, 2013.

[45] HSA Platform System Architecture Specification Version 1.O. Tech. rep. HSA foundation, 2015.

[46] N. Sakharnykh. Everything You Need to Know About Unified Memory. http : / / on-demand. gputechconf . com / gtc / 2018 / presentation / s8430-everything-you-need-to-know-about-unified-memory . pdf, retrieved in Oct. 2018. NVIDIA.

[47] J. D. Owens, D. Luebke, N. Govindaraju, M. Harris, J. Krüger, A. E. Lefohn, and T. J. Purcell. "A Survey of general-purpose computation on graphics hardware.” In: Computer Graphics Forum 26.1 (Mar. 2007), pp. 80-113.

[48] Q. Li, C. Zhong, K. Zhao, X. Mei, and X. Chu. "Implementation and analysis of AES encryption on GPU." In: IEEE International Conference on High Performance Computing and Communication, International Conference on Embedded Software and Systems. June 2012, pp. 843-848.

[49] S. Huang, S. Xiao, and W. Feng. "On the energy efficiency of graphics processing units for scientific computing." In: IEEE International Symposium on Parallel Distributed Processing. May 2009, pp. 1-8.

[50] X. Ma, M. Dong, L. Zhong, and Z. Deng. "Statistical power consumption analysis and modeling for GPU-based computing." In: Proceedings of ACM SOSP Workshop on Power Aware Computing and Systems (HotPower). 2009, pp. 1-5.

[51] A. Betts, N. Chong, A. Donaldson, S. Qadeer, and P. Thomson. "GPUVerify: A verifier for GPU kernels.” In: ACM SIGPLAN Notices 47.10 (Oct. 2012), pp. 113-132.

[52] N. Agarwal, D. Nellans, M. Stephenson, M. O'Connor, and S. W. Keckler. "Page placement strategies for GPUs within heterogeneous memory systems." In: ACM SIGPLAN Notices 50.4 (Mar. 2015), pp. 607618.

[53] K. Gupta and J. D. Owens. "Compute and memory optimizations for high-quality speech recognition on low-end GPU processors." In: International Conference on High Performance Computing. Dec. 2011, pp. 1-10.

[54] M. Daga, A. M. Aji, and W. Feng. "On the efficacy of a fused CPU+GPU processor (or APU) for parallel computing." In: Symposium on Application Accelerators in High-Performance Computing. July 2011, pp. 141-149. 
[55] S. Mu, C. Wang, M. Liu, D. Li, M. Zhu, X. Chen, X. Xie, and Y. Deng. "Evaluating the potential of graphics processors for high performance embedded computing." In: Design, Automation and Test in Europe Conference (DATE). Mar. 2011, pp. 1-6.

[56] J. Leskela, J. Nikula, and M. Salmela. "OpenCL embedded profile prototype in mobile device.” In: IEEE Workshop on Signal Processing Systems. Oct. 2009, pp. 279-284.

[57] N. Singhal, I. K. Park, and S. Cho. "Implementation and optimization of image processing algorithms on handheld GPU.” In: IEEE International Conference on Image Processing. Sept. 2010, pp. 4481-4484.

[58] K. Cheng and Y. Wang. "Using mobile GPU for general-purpose computing - a case study of face recognition on smartphones." In: Proceedings of International Symposium on VLSI Design, Automation and Test. Apr. 2011, pp. 1-4.

[59] G. A. Elliott and J. H. Anderson. "Real-world constraints of GPUs in real-time systems." In: IEEE International Conference on Embedded and Real-Time Computing Systems and Applications. Aug. 2011, pp. 48-54.

[60] M. Lindgren, K. Sandström, T. Nolte, and D. Hallmans. "Applicability of using internal GPGPUs in industrial control systems.” In: Proceedings of IEEE Emerging Technology and Factory Automation (ETFA). Sept. 2014, pp. 1-7.

[61] S. Kato, K. Lakshmanan, R. Rajkumar, and Y. Ishikawa. "TimeGraph: GPU scheduling for real-time multi-tasking environments.” In: Proceedings of USENIX Conference on USENIX Annual Technical Conference. June 2011, pp. 1-14.

[62] G. A. Elliott and J. H. Anderson. "Globally scheduled real-time multiprocessor systems with GPUs.” In: Real-Time Systems 48.1 (Jan. 2012), pp. 34-74.

[63] G. A. Elliott, B. C. Ward, and J. H. Anderson. "GPUSync: A framework for real-time GPU management.” In: IEEE Real-Time Systems Symposium. Dec. 2013, pp. 33-44.

[64] S. Kato, K. Lakshmanan, A. Kumar, M. Kelkar, Y. Ishikawa, and R. Rajkumar. "RGEM: A responsive GPGPU execution model for runtime engines." In: IEEE Real-Time Systems Symposium. Nov. 2011, pp. 5766.

[65] G. A. Elliott and J. H. Anderson. "Robust real-time multiprocessor interrupt handling motivated by GPUs.” In: Euromicro Conference on Real-Time Systems. July 2012, pp. 267-276. 
[66] H. Lee and M. A. A. Faruque. "GPU-EvR: Run-time event based realtime scheduling framework on GPGPU platform." In: Design, Automation and Test in Europe Conference (DATE). Mar. 2014, pp. 1-6.

[67] U. Verner, A. Schuster, and M. Silberstein. "Processing data streams with hard real-time constraints on heterogeneous systems." In: Proceedings of the International Conference on Supercomputing. May 2011, pp. 120-129.

[68] U. Verner, A. Schuster, M. Silberstein, and A. Mendelson. "Scheduling processing of real-time data streams on heterogeneous multi-GPU systems." In: Proceedings of the Annual International Systems and Storage Conference. June 2012, 8:1-8:12.

[69] A. Horga, S. Chattopadhyay, P. Eles, and Z. Peng. "Measurement based execution time analysis of GPGPU programs via SE+GA." In: Euromicro Conference on Digital System Design (DSD). Aug. 2018, pp. 30-37.

[70] K. Berezovskyi, K. Bletsas, and B. Andersson. "Makespan computation for GPU threads running on a single streaming multiprocessor." In: Euromicro Conference on Real-Time Systems. July 2012, pp. 277286.

[71] A. Betts and A. Donaldson. "Estimating the WCET of GPUaccelerated applications using hybrid analysis." In: Euromicro Conference on Real-Time Systems. July 2013, pp. 193-202.

[72] S. Chattopadhyay, C. L. Kee, A. Roychoudhury, T. Kelter, P. Marwedel, and H. Falk. "A unified WCET analysis framework for multi-core platforms." In: IEEE Real Time and Embedded Technology and Applications Symposium. Apr. 2012, pp. 99-108.

[73] M. H. Santriaji and H. Hoffmann. "MERLOT: Architectural support for energy-efficient real-time processing in GPUs." In: IEEE Real-Time and Embedded Technology and Applications Symposium (RTAS). Apr. 2018, pp. 214-226.

[74] H. Lee and M. A. A. Faruque. "Run-time scheduling framework for event-driven applications on a GPU-based embedded system." In: IEEE Transactions on Computer-Aided Design of Integrated Circuits and Systems 35.12 (Dec. 2016), pp. 1956-1967.

[75] B. Dietrich and S. Chakraborty. "Forget the battery, let's play games!" In: IEEE Symposium on Embedded Systems for Real-time Multimedia (ESTIMedia). Oct. 2014, pp. 1-8.

[76] B. Dietrich and S. Chakraborty. "Lightweight graphics instrumentation for game state-specific power management in Android." In: Multimedia Systems 20.5 (Oct. 2014), pp. 563-578. 
[77] A. Pathania, Q. Jiao, A. Prakash, and T. Mitra. "Integrated CPU-GPU power management for 3D mobile games." In: ACM/EDAC/IEEE Design Automation Conference (DAC). June 2014, pp. 1-6.

[78] A. Pathania, A. E. Irimiea, A. Prakash, and T. Mitra. "Powerperformance modelling of mobile gaming workloads on heterogeneous MPSoCs." In: ACM/EDAC/IEEE Design Automation Conference (DAC). June 2015, pp. 1-6.

[79] C. Hwang, S. Pushp, C. Koh, J. Yoon, Y. Liu, S. Choi, and J. Song. "RAVEN: Perception-aware optimization of power consumption for mobile games." In: Proceedings of the Annual International Conference on Mobile Computing and Networking. Oct. 2017, pp. 422-434.

[80] J.-M. Arnau, J.-M. Parcerisa, and P. Xekalakis. "TEAPOT: A toolset for evaluating performance, power and image quality on mobile graphics systems." In: International ACM Conference on International Conference on Supercomputing. 2013, pp. 37-46.

[81] S. He, Y. Liu, and H. Zhou. "Optimizing smartphone power consumption through dynamic resolution scaling." In: Proceedings of the Annual International Conference on Mobile Computing and Networking. Sept. 2015, pp. 27-39.

[82] J. Pool, A. Lastra, and M. Singh. "Energy-precision tradeoffs in mobile Graphics Processing Units.” In: 2008 IEEE International Conference on Computer Design. Oct. 2008, pp. 60-67.

[83] K. Yan, X. Zhang, J. Tan, and X. Fu. "Redefining QoS and customizing the power management policy to satisfy individual mobile users." In: The Annual IEEE/ACM International Symposium on Microarchitecture. 2016, 53:1-53:12.

[84] C. Xie, X. Fu, and S. Song. "Perception-oriented 3D rendering approximation for modern graphics processors." In: IEEE International Symposium on High Performance Computer Architecture (HPCA). Feb. 2018, pp. 362-374.

[85] A. S. Bischoff. "User-experience-aware system optimisation for mobile systems." PhD thesis. University of Southampton, 2016.

[86] A. Devlic. "On optimization of quality of user experience and wireless network bandwidth in video content delivery." PhD thesis. KTH, Mobile Service Laboratory (MS Lab), 2015.

[87] S. Han, K. Jang, K. Park, and S. Moon. "PacketShader: A GPUaccelerated Software Router." In: SIGCOMM Computer Communication Review 40.4 (Aug. 2010), pp. 195-206.

[88] Y. Li, D. Zhang, A. X. Liu, and J. Zheng. "GAMT: A fast and scalable IP lookup engine for GPU-based software routers.” In: Architectures for Networking and Communications Systems. Oct. 2013, pp. 1-12. 
[89] Y. Zhu, Y. Deng, and Y. Chen. "Hermes: An integrated CPU/GPU microarchitecture for IP routing." In: ACM/EDAC/IEEE Design Automation Conference (DAC). June 2011, pp. 1044-1049.

[90] W. Sun and R. Ricci. "Fast and flexible: Parallel packet processing with GPUs and click." In: Architectures for Networking and Communications Systems. Oct. 2013, pp. 25-35.

[91] P. Gupta and N. McKeown. "Algorithms for packet classification." In: IEEE Network 15.2 (Mar. 2001), pp. 24-32.

[92] S. Zhou, S. G. Singapura, and V. K. Prasanna. "High-performance packet classification on GPU." In: IEEE High Performance Extreme Computing Conference (HPEC). Sept. 2014, pp. 1-6.

[93] M. Varvello, R. Laufer, F. Zhang, and T. V. Lakshman. "Multilayer Packet Classification With Graphics Processing Units.” In: IEEE/ACM Transactions on Networking 24.5 (Oct. 2016), pp. 2728-2741.

[94] K. Kang and Y. S. Deng. "Scalable packet classification via GPU metaprogramming." In: Design, Automation and Test in Europe Conference (DATE). Mar. 2011, pp. 1-4.

[95] A. Kalia, D. Zhou, M. Kaminsky, and D. G. Andersen. "Raising the bar for using GPUs in software packet processing." In: Proceedings of the USENIX Conference on Networked Systems Design and Implementation. Sept. 2015, pp. 409-423.

[96] S. Yu and P. Y. Chen. "Emerging memory technologies: recent trends and prospects." In: IEEE Solid-State Circuits Magazine 8.2 (Mar. 2016), pp. 43-56.

[97] Y. Xie. "Future memory and interconnect technologies." In: Design, Automation and Test in Europe Conference (DATE). Mar. 2013, pp. 964-969.

[98] Y. Tian, S. Puthoor, J. L. Greathouse, B. M. Beckmann, and D. A. Jiménez. "Adaptive GPU cache bypassing." In: Proceedings of the Workshop on General Purpose Processing Using GPUs. Feb. 2015, pp. 25-35.

[99] C. Nugteren, G. van den Braak, H. Corporaal, and H. Bal. "A detailed GPU cache model based on reuse distance theory." In: IEEE International Symposium on High Performance Computer Architecture (HPCA). Feb. 2014, pp. 37-48.

[100] M. M. Baskaran and R. Bordawekar. Optimizing sparse matrix-vector multiplication on GPUs. IBM Research Report RC24704. Apr. 2009.

[101] F. Bistaffa, A. Farinelli, and N. Bombieri. "Optimising memory management for belief propagation in junction trees using GPGPUs." In: IEEE International Conference on Parallel and Distributed Systems (ICPADS). Dec. 2014, pp. 526-533. 
[102] P. S. Rawat, C. Hong, M. Ravishankar, V. Grover, L. N. Pouchet, A. Rountev, and P. Sadayappan. "Resource conscious reuse-driven tiling for GPUs.” In: International Conference on Parallel Architecture and Compilation Techniques (PACT). Sept. 2016, pp. 99-111.

[103] L. Wang, W. Wu, Z. Xu, J. Xiao, and Y. Yang. "BLASX: a high performance level-3 BLAS library for heterogeneous multi-GPU computing." In: International Conference on Supercomputing (ICS). June 2016, 20:1-20:11.

[104] A. Li, G. van den Braak, A. Kumar, and H. Corporaal. "Adaptive and transparent cache bypassing for GPUs." In: Proceedings of the International Conference for High Performance Computing, Networking, Storage and Analysis. Nov. 2015, pp. 1-12.

[105] S. Mittal. “A Survey Of Cache Bypassing Techniques.” In: MDPI Journal of Low Power Electronics and Applications (Special issue) 6 (2016), $5: 1-5: 30$.

[106] A. Li, S. L. Song, W. Liu, X. Liu, A. Kumar, and H. Corporaal. "Localityaware CTA clustering for modern GPUs." In: Proceedings of the International Conference on Architectural Support for Programming Languages and Operating Systems. Apr. 2017, pp. 297-311.

[107] Y. Liang and X. Li. "Efficient kernel management on GPUs." In: $A C M$ Transactions on Embedded Computing Systems (TECS) 16.4 (May 2017), 115:1-115:24. 


\section{Department of Computer and Information Science Linköping University}

\section{Dissertations}

\section{Linköping Studies in Science and Technology}

No. 14 Anders Haraldsson: A Program Manipulation System Based on Partial Evaluation, 1977, ISBN 91-7372-144-1.

No. 17 Bengt Magnhagen: Probability Based Verification of Time Margins in Digital Designs, 1977, ISBN 91-7372-157-3.

No. 18 Mats Cedwall: Semantisk analys av processbeskrivningar i naturligt språk, 1977 ISBN 91-7372-168-9.

No. 22 Jaak Urmi: A Machine Independent LISP Compiler and its Implications for Ideal Hardware, 1978, ISBN 91-7372-188-3.

No. 33 Tore Risch: Compilation of Multiple File Queries in a Meta-Database System, 1978, ISBN 91-7372-232-4.

No. 51 Erland Jungert: Synthesizing Database Structures from a User Oriented Data Model, 1980, ISBN 91-7372-387-8.

No. 54 Sture Hägglund: Contributions to the Development of Methods and Tools for Interactive Design of Applications Software, 1980, ISBN 91-7372-404-1.

No. 55 Pär Emanuelson: Performance Enhancement in a Well-Structured Pattern Matcher through Partial Evaluation, 1980, ISBN 91-7372-403-3.

No. 58 Bengt Johnsson, Bertil Andersson: The Human-Computer Interface in Commercial Systems, 1981, ISBN 91-7372-414-9.

No. 69 H. Jan Komorowski: A Specification of an Abstract Prolog Machine and its Application to Partial Evaluation, 1981, ISBN 91-7372-479-3.

No. 71 René Reboh: Knowledge Engineering Techniques and Tools for Expert Systems, 1981, ISBN 91-7372-489-0.

No. 77 Östen Oskarsson: Mechanisms of Modifiability in large Software Systems, 1982, ISBN 91-7372-527-7.

No. 94 Hans Lunell: Code Generator Writing Systems, 1983 ISBN 91-7372-652-4

No. 97 Andrzej Lingas: Advances in Minimum Weight Triangulation, 1983, ISBN 91-7372-660-5.

No. 109 Peter Fritzson: Towards a Distributed Programming Environment based on Incremental Compilation, 1984, ISBN 91-7372-801-2

No. 111 Erik Tengvald: The Design of Expert Planning Systems. An Experimental Operations Planning System for Turning, 1984, ISBN 91-7372-805-5.

No. 155 Christos Levcopoulos: Heuristics for Minimum Decompositions of Polygons, 1987, ISBN 91-7870-133-3.

No. 165 James W. Goodwin: A Theory and System for Non-Monotonic Reasoning, 1987, ISBN 91-7870-183-X.

No. 170 Zebo Peng: A Formal Methodology for Automated Synthesis of VLSI Systems, 1987, ISBN 91-7870-225-9.

No. 174 Johan Fagerström: A Paradigm and System for Design of Distributed Systems, 1988, ISBN 91-7870-301-8.

No. 192 Dimiter Driankov: Towards a Many Valued Logic of Quantified Belief, 1988, ISBN 91-7870-374-3.

No. 213 Lin Padgham: Non-Monotonic Inheritance for an Object Oriented Knowledge Base, 1989 , ISBN 91-7870-485-5.

No. 214 Tony Larsson: A Formal Hardware Description and
Verification Method, 1989, ISBN 91-7870-517-7.

No. 221 Michael Reinfrank: Fundamentals and Logical Foundations of Truth Maintenance, 1989 ISBN 91-7870-546-0.

No. 239 Jonas Löwgren: Knowledge-Based Design Support and Discourse Management in User Interface Management Systems, 1991, ISBN 91-7870-720-X.

No. 244 Henrik Eriksson: Meta-Tool Support for Knowledge Acquisition, 1991, ISBN 91-7870-746-3.

No. 252 Peter Eklund: An Epistemic Approach to Interactive Design in Multiple Inheritance Hierarchies, 1991, ISBN 91-7870-784-6.

No. 258 Patrick Doherty: NML3 - A Non-Monotonic Formalism with Explicit Defaults, 1991 ISBN 91-7870-816-8.

No. 260 Nahid Shahmehri: Generalized Algorithmic Debugging, 1991, ISBN 91-7870-828-1.

No. 264 Nils Dahlbäck: Representation of Discourse-Cognitive and Computational Aspects, 1992, ISBN 91-7870-850-8.

No. 265 Ulf Nilsson: Abstract Interpretations and Abstract Machines: Contributions to a Methodology for the Implementation of Logic Programs, 1992, ISBN 91-7870-858-3.

No. 270 Ralph Rönnquist: Theory and Practice of Tense-bound Object References, 1992 ISBN 91-7870-873-7.

No. 273 Björn Fjellborg: Pipeline Extraction for VLSI Data Path Synthesis, 1992, ISBN 91-7870-880-X

No. 276 Staffan Bonnier: A Formal Basis for Horn Clause Logic with External Polymorphic Functions, 1992, ISBN 91-7870-896-6.

No. 277 Kristian Sandahl: Developing Knowledge Management Systems with an Active Expert Methodology, 1992, ISBN 91-7870-897-4.

No. 281 Christer Bäckström: Computational Complexity of Reasoning about Plans, 1992, ISBN 91-7870-979-2.

No. 292 Mats Wirén: Studies in Incremental Natural Language Analysis, 1992, ISBN 91-7871-027-8.

No. 297 Mariam Kamkar: Interprocedural Dynamic Slicing with Applications to Debugging and Testing, 1993, ISBN 91-7871-065-0.

No. 302 Tingting Zhang: A Study in Diagnosis Using Classification and Defaults, 1993 , ISBN 91-7871-078-2.

No. 312 Arne Jönsson: Dialogue Management for Natural Language Interfaces - An Empirical Approach, 1993, ISBN 91-7871-110-X.

No. 338 Simin Nadjm-Tehrani: Reactive Systems in Physical Environments: Compositional Modelling and Framework for Verification, 1994 ISBN 91-7871-237-8.

No. 371 Bengt Savén: Business Models for Decision Support and Learning. A Study of Discrete-Event Manufacturing Simulation at Asea/ABB 1968-1993, 1995, ISBN 91-7871-494-X.

No. 375 Ulf Söderman: Conceptual Modelling of Mode Switching Physical Systems, 1995 ISBN 91-7871-516-4.

No. 383 Andreas Kågedal: Exploiting Groundness in Logic Programs, 1995, ISBN 91-7871-538-5.

No. 396 George Fodor: Ontological Control, Description, Identification and Recovery from Problematic Control Situations, 1995, ISBN 91-7871-603-9.

No. 413 Mikael Pettersson: Compiling Natural Semantics, 
1995, ISBN 91-7871-641-1.

No. 414 Xinli Gu: RT Level Testability Improvement by Testability Analysis and Transformations, 1996, ISBN 91-7871-654-3.

No. 416 Hua Shu: Distributed Default Reasoning, 1996, ISBN 91-7871-665-9.

No. 429 Jaime Villegas: Simulation Supported Industrial Training from an Organisational Learning Perspective - Development and Evaluation of the SSIT Method, 1996, ISBN 91-7871-700-0.

No. 431 Peter Jonsson: Studies in Action Planning: Algorithms and Complexity, 1996, ISBN 91-7871-704-3.

No. 437 Johan Boye: Directional Types in Logic Programming, 1996, ISBN 91-7871-725-6.

No. 439 Cecilia Sjöberg: Activities, Voices and Arenas: Participatory Design in Practice, 1996, ISBN 91-7871-728-0.

No. 448 Patrick Lambrix: Part-Whole Reasoning in Description Logics, 1996, ISBN 91-7871-820-1.

No. 452 Kjell Orsborn: On Extensible and Object-Relational Database Technology for Finite Element Analysis Applications, 1996, ISBN 91-7871-827-9.

No. 459 Olof Johansson: Development Environments for Complex Product Models, 1996, ISBN 91-7871-855-4.

No. 461 Lena Strömbäck: User-Defined Constructions in Unification-Based Formalisms, 1997, ISBN 91-7871-857-0.

No. 462 Lars Degerstedt: Tabulation-based Logic Programming: A Multi-Level View of Query Answering, 1996, ISBN 91-7871-858-9.

No. 475 Fredrik Nilsson: Strategi och ekonomisk styrning En studie av hur ekonomiska styrsystem utformas och används efter företagsförvärv, 1997, ISBN 91-7871-914-3.

No. 480 Mikael Lindvall: An Empirical Study of Requirements-Driven Impact Analysis in Object-Oriented Software Evolution, 1997, ISBN 91-7871-927-5.

No. 485 Göran Forslund: Opinion-Based Systems: The Cooperative Perspective on Knowledge-Based Decision Support, 1997, ISBN 91-7871-938-0.

No. 494 Martin Sköld: Active Database Management Systems for Monitoring and Control, 1997, ISBN 91-7219-002-7.

No. 495 Hans Olsén: Automatic Verification of Petri Nets in a CLP framework, 1997, ISBN 91-7219-011-6.

No. 498 Thomas Drakengren: Algorithms and Complexity for Temporal and Spatial Formalisms, 1997, ISBN 91-7219-019-1.

No. 502 Jakob Axelsson: Analysis and Synthesis of Heterogeneous Real-Time Systems, 1997, ISBN 91-7219-035-3.

No. 503 Johan Ringström: Compiler Generation for Data-Parallel Programming Languages from Two-Level Semantics Specifications, 1997 ISBN 91-7219-045-O.

No. 512 Anna Moberg: Närhet och distans - Studier av kommunikationsmönster i satellitkontor och flexibla kontor, 1997, ISBN 91-7219-119-8.

No. 520 Mikael Ronström: Design and Modelling of a Parallel Data Server for Telecom Applications, 1998, ISBN 91-7219-169-4.

No. 522 Niclas Ohlsson: Towards Effective Fault Prevention An Empirical Study in Software Engineering, 1998, ISBN 91-7219-176-7.

No. 526 Joachim Karlsson: A Systematic Approach for Prioritizing Software Requirements, 1998, ISBN 91-7219-184-8.

No. 530 Henrik Nilsson: Declarative Debugging for Lazy Functional Languages, 1998, ISBN 91-7219-197-X.

No. 555 Jonas Hallberg: Timing Issues in High-Level Synthesis, 1998, ISBN 91-7219-369-7.
No. 561 Ling Lin: Management of 1-D Sequence Data - From Discrete to Continuous, 1999, ISBN 91-7219-402-2.

No. 563 Eva L Ragnemalm: Student Modelling based on Collaborative Dialogue with a Learning Companion, 1999, ISBN 91-7219-412-X

No. 567 Jörgen Lindström: Does Distance matter? On geographical dispersion in organisations, 1999, ISBN 91-7219-439-1.

No. 582 Vanja Josifovski: Design, Implementation and Evaluation of a Distributed Mediator System for Data Integration, 1999, ISBN 91-7219-482-0.

No.589 Rita Kovordányi: Modeling and Simulating Inhibitory Mechanisms in Mental Image Reinterpretation - Towards Cooperative Human-Computer Creativity, 1999 , ISBN 91-7219-506-1.

No. 592 Mikael Ericsson: Supporting the Use of Design Knowledge - An Assessment of Commenting Agents, 1999, ISBN 91-7219-532-0.

No. 593 Lars Karlsson: Actions, Interactions and Narratives, 1999, ISBN 91-7219-534-7.

No. 594 C. G. Mikael Johansson: Social and Organizational Aspects of Requirements Engineering Methods - A practice-oriented approach, 1999, ISBN 91-7219-541-X.

No. 595 Jörgen Hansson: Value-Driven Multi-Class Overload Management in Real-Time Database Systems, 1999, ISBN 91-7219-542-8.

No. 596 Niklas Hallberg: Incorporating User Values in the Design of Information Systems and Services in the Public Sector: A Methods Approach, 1999, ISBN 91-7219-543-6.

No. 597 Vivian Vimarlund: An Economic Perspective on the Analysis of Impacts of Information Technology: From Case Studies in Health-Care towards General Models and Theories, 1999, ISBN 91-7219-544-4.

No. 598 Johan Jenvald: Methods and Tools in Computer-Supported Taskforce Training, 1999, ISBN 91-7219-547-9.

No. 607 Magnus Merkel: Understanding and enhancing translation by parallel text processing, 1999 , ISBN 91-7219-614-9.

No. 611 Silvia Coradeschi: Anchoring symbols to sensory data, 1999, ISBN 91-7219-623-8.

No. 613 Man Lin: Analysis and Synthesis of Reactive Systems: A Generic Layered Architecture Perspective, 1999, ISBN 91-7219-630-0.

No. 618 Jimmy Tjäder: Systemimplementering i praktiken En studie av logiker i fyra projekt, 1999, ISBN 91-7219-657-2.

No. 627 Vadim Engelson: Tools for Design, Interactive Simulation, and Visualization of Object-Oriented Models in Scientific Computing, 2000, ISBN 91-7219-709-9.

No. 637 Esa Falkenroth: Database Technology for Control and Simulation, 2000, ISBN 91-7219-766-8.

No. 639 Per-Arne Persson: Bringing Power and Knowledge Together: Information Systems Design for Autonomy and Control in Command Work, 2000, ISBN 91-7219-796-X.

No. 660 Erik Larsson: An Integrated System-Level Design for Testability Methodology, 2000, ISBN 91-7219-890-7.

No. 688 Marcus Bjäreland: Model-based Execution Monitoring, 2001, ISBN 91-7373-016-5.

No. 689 Joakim Gustafsson: Extending Temporal Action Logic, 2001, ISBN 91-7373-017-3.

No. 720 Carl-Johan Petri: Organizational Information Provision - Managing Mandatory and Discretionary Use of Information Technology, 2001, ISBN 91-7373-126-9.

No. 724 Paul Scerri: Designing Agents for Systems with Adjustable Autonomy, 2001, ISBN 91-7373-207-9.

No. 725 Tim Heyer: Semantic Inspection of Software 
Artifacts: From Theory to Practice, 2001, ISBN 91-7373-208-7.

No. 726 Pär Carlshamre: A Usability Perspective on Requirements Engineering - From Methodology to Product Development, 2001, ISBN 91-7373-212-5.

No. 732 Juha Takkinen: From Information Management to Task Management in Electronic Mail, 2002, ISBN 91-7373-258-3.

No. 745 Johan Åberg: Live Help Systems: An Approach to Intelligent Help for Web Information Systems, 2002, ISBN 91-7373-311-3.

No. 746 Rego Granlund: Monitoring Distributed Teamwork Training, 2002, ISBN 91-7373-312-1.

No. 747 Anneli Hagdahl: Development of IT-supported Interorganisational Collaboration - A Case Study in the Swedish Public Sector, 2002, ISBN 91-7373-314-8.

No. 749 Sofie Pilemalm: Information Technology for Non-Profit Organisations - Extended Participatory Design of an Information System for Trade Union Shop Stewards, 2002, ISBN 91-7373-318-0.

No. 757 Henrik André-Jönsson: Indexing Strategies for Time Series Data, 2002, ISBN 917373-346-6.

No. 758 Erik Berglund: Library Communication Among Programmers Worldwide, 2002, ISBN 91-7373-349-0.

No. 765 Stefan Holmlid: Adapting users: Towards a theory of use quality, 2002, ISBN 91-7373-397-0

No. 771 Magnus Morin: Multimedia Representations of Distributed Tactical Operations, 2002, ISBN 91-7373-421-7.

No. 772 Pawel Pietrzak: A Type-Based Framework for Locating Errors in Constraint Logic Programs, 2002, ISBN 91-7373-422-5.

No. 774 Choong-ho Yi: Modelling Object-Oriented Dynamic Systems Using a Logic-Based Framework, 2002, ISBN 91-7373-424-1

No. 779 Mathias Broxvall: A Study in the Computational Complexity of Temporal Reasoning, 2002, ISBN 91-7373-440-3.

No. 785 Lars Hult: Publika Informationstjänster. En studie av den Internetbaserade encyklopedins bruksegenskaper, 2003, ISBN 91-7373-461-6.

No. 793 Asmus Pandikow: A Generic Principle for Enabling Interoperability of Structured and Object-Oriented Analysis and Design Tools, 2002, ISBN 91-7373-479-9.

No. 800 Lars Taxén: A Framework for the Coordination of Complex Systems' Development, 2003, ISBN 91-7373-604-X.

No. 808 Klas Gäre: Tre perspektiv på förväntningar och förändringar i samband med införande av informationssystem, 2003, ISBN 91-7373-618-X.

No. 821 Mikael Kindborg: Concurrent Comics programming of social agents by children, 2003, ISBN 91-7373-651-1.

No. 823 Christina Ölvingson: On Development of Information Systems with GIS Functionality in Public Health Informatics: A Requirements Engineering Approach, 2003, ISBN 91-7373-656-2.

No. 828 Tobias Ritzau: Memory Efficient Hard Real-Time Garbage Collection, 2003, ISBN 91-7373-666-X.

No. 833 Paul Pop: Analysis and Synthesis of Communication-Intensive Heterogeneous Real-Time Systems, 2003, ISBN 91-7373-683-X.

No. 852 Johan Moe: Observing the Dynamic Behaviour of Large Distributed Systems to Improve Development and Testing - An Empirical Study in Software Engineering, 2003, ISBN 91-7373-779-8.

No. 867 Erik Herzog: An Approach to Systems Engineering Tool Data Representation and Exchange, 2004, ISBN 91-7373-929-4.

No. 869 Jo Skåmedal: Telecommuting's Implications on Travel and Travel Patterns, 2004,
ISBN 91-7373-935-9.

No. 870 Linda Askenäs: The Roles of IT - Studies of Organising when Implementing and Using Enterprise Systems, 2004, ISBN 91-7373-936-7.

No. 872 Aseel Berglund: Augmenting the Remote Control: Studies in Complex Information Navigation for Digital TV, 2004, ISBN 91-7373-940-5.

No. 873 Peter Bunus: Debugging Techniques for Equation-Based Languages, 2004, ISBN 91-7373-941-3.

No. 874 Annika Flycht-Eriksson: Design and Use of Ontologies in Information-Providing Dialogue Systems, 2004, ISBN 91-7373-947-2.

No. 876 Jonas Mellin: Resource-Predictable and Efficient Monitoring of Events, 2004, ISBN 91-7373-956-1.

No. 882 Robert Eklund: Disfluency in Swedish human-human and human-machine travel booking dialogues, 2004, ISBN 91-7373-966-9.

No. 883 Magnus Bång: Computing at the Speed of Paper: Ubiquitous Computing Environments for Healthcare Professionals, 2004, ISBN 91-7373-971-5.

No. 887 Anders Lindström: English and other Foreign Linguistic Elements in Spoken Swedish. Studies of Productive Processes and their Modelling using Finite-State Tools, 2004, ISBN 91-7373-981-2.

No. 889 Zhiping Wang: Capacity-Constrained Production-inventory systems - Modelling and Analysis in both a traditional and an e-business context, 2004, ISBN 91-85295-08-6.

No. 893 Pernilla Qvarfordt: Eyes on Multimodal Interaction, 2004, ISBN 91-85295-30-2.

No. 900 Mattias Arvola: Shades of use: The dynamics of interaction design for sociable use, 2004, ISBN 91-85295-42-6.

No. 910 Magnus Kald: In the Borderland between Strategy and Management Control - Theoretical Framework and Empirical Evidence, 2004, ISBN 91-85295-82-5.

No. 918 Jonas Lundberg: Shaping Electronic News: Genre Perspectives on Interaction Design, 2004, ISBN 91-85297-14-3.

No. 920 Luis Alejandro Cortés: Verification and Scheduling Techniques for Real-Time Embedded Systems, 2004, ISBN 91-85297-21-6.

No. 929 Diana Szentivanyi: Performance Studies of Fault-Tolerant Middleware, 2005, ISBN 91-85297-58-5.

No. 933 Mikael Cäker: Management Accounting as Constructing and Opposing Customer Focus: Three Case Studies on Management Accounting and Customer Relations, 2005, ISBN 91-85297-64-X.

No. 937 Jonas Kvarnström: TALplanner and Other Extensions to Temporal Action Logic, 2005, ISBN 91-85297-75-5.

No. 938 Bourhane Kadmiry: Fuzzy Gain-Scheduled Visual Servoing for Unmanned Helicopter, 2005 ISBN 91-85297-76-3.

No. 945 Gert Jervan: Hybrid Built-In Self-Test and Test Generation Techniques for Digital Systems, 2005 ISBN 91-85297-97-6.

No. 946 Anders Arpteg: Intelligent Semi-Structured Information Extraction, 2005, ISBN 91-85297-98-4.

No. 947 Ola Angelsmark: Constructing Algorithms for Constraint Satisfaction and Related Problems Methods and Applications, 2005, ISBN 91-85297-99-2.

No. 963 Calin Curescu: Utility-based Optimisation of Resource Allocation for Wireless Networks, 2005, ISBN 91-85457-07-8

No. 972 Björn Johansson: Joint Control in Dynamic Situations, 2005, ISBN 91-85457-31-0.

No. 974 Dan Lawesson: An Approach to Diagnosability Analysis for Interacting Finite State Systems, 2005, ISBN 91-85457-39-6. 
No. 979 Claudiu Duma: Security and Trust Mechanisms for Groups in Distributed Services, 2005 ISBN 91-85457-54-X.

No. 983 Sorin Manolache: Analysis and Optimisation of Real-Time Systems with Stochastic Behaviour, 2005, ISBN 91-85457-60-4

No. 986 Yuxiao Zhao: Standards-Based Application Integration for Business-to-Business Communications, 2005, ISBN 91-85457-66-3

No. 1004 Patrik Haslum: Admissible Heuristics for Automated Planning, 2006, ISBN 91-85497-28-2.

No. 1005 Aleksandra Tešanovic: Developing Reusable and Reconfigurable Real-Time Software using Aspects and Components, 2006, ISBN 91-85497-29-0.

No. 1008 David Dinka: Role, Identity and Work: Extending the design and development agenda, 2006, ISBN 91-85497-42-8.

No. 1009 Iakov Nakhimovski: Contributions to the Modeling and Simulation of Mechanical Systems with Detailed Contact Analysis, 2006, ISBN 91-85497-43-X.

No. 1013 Wilhelm Dahllöf: Exact Algorithms for Exact Satisfiability Problems, 2006, ISBN 91-85523-97-6.

No. 1016 Levon Saldamli: PDEModelica - A High-Level Language for Modeling with Partial Differential Equations, 2006, ISBN 91-85523-84-4.

No. 1017 Daniel Karlsson: Verification of Component-based Embedded System Designs, 2006, ISBN 91-85523-79-8.

No. 1018 Ioan Chisalita: Communication and Networking Techniques for Traffic Safety Systems, 2006 ISBN 91-85523-77-1.

No. 1019 Tarja Susi: The Puzzle of Social Activity - The Significance of Tools in Cognition and Cooperation, 2006, ISBN 91-85523-71-2

No. 1021 Andrzej Bednarski: Integrated Optimal Code Generation for Digital Signal Processors, 2006, ISBN 91-85523-69-0.

No. 1022 Peter Aronsson: Automatic Parallelization of Equation-Based Simulation Programs, 2006, ISBN 91-85523-68-2.

No. 1030 Robert Nilsson: A Mutation-based Framework for Automated Testing of Timeliness, 2006 ISBN 91-85523-35-6.

No. 1034 Jon Edvardsson: Techniques for Automatic Generation of Tests from Programs and Specifications, 2006, ISBN 91-85523-31-3.

No. 1035 Vaida Jakoniene: Integration of Biological Data, 2006, ISBN 91-85523-28-3.

No. 1045 Genevieve Gorrell: Generalized Hebbian Algorithms for Dimensionality Reduction in Natural Language Processing, 2006, ISBN 91-85643-88-2.

No. 1051 Yu-Hsing Huang: Having a New Pair of Glasses Applying Systemic Accident Models on Road Safety, 2006, ISBN 91-85643-64-5.

No. 1054 Åsa Hedenskog: Perceive those things which cannot be seen - A Cognitive Systems Engineering perspective on requirements management, 2006, ISBN 91-85643-57-2.

No. 1061 Cécile Åberg: An Evaluation Platform for Semantic Web Technology, 2007, ISBN 91-85643-31-9.

No. 1073 Mats Grindal: Handling Combinatorial Explosion in Software Testing, 2007, ISBN 978-91-85715-74-9.

No. 1075 Almut Herzog: Usable Security Policies for Runtime Environments, 2007, ISBN 978-91-85715-65-7.

No. 1079 Magnus Wahlström: Algorithms, measures, and upper bounds for Satisfiability and related problems, 2007, ISBN 978-91-85715-55-8.

No. 1083 Jesper Andersson: Dynamic Software Architectures, 2007, ISBN 978-91-85715-46-6.

No. 1086 Ulf Johansson: Obtaining Accurate and Compre-hensible Data Mining Models - An Evolutionary Approach, 2007, ISBN 978-91-85715-34-3.
No. 1089 Traian Pop: Analysis and Optimisation of Distributed Embedded Systems with Heterogeneou Scheduling Policies, 2007, ISBN 978-91-85715-27-5.

No. 1091 Gustav Nordh: Complexity Dichotomies for CSP-related Problems, 2007, ISBN 978-91-85715-20-6.

No. 1106 Per Ola Kristensson: Discrete and Continuous Shape Writing for Text Entry and Control, 2007, ISBN 978-91-85831-77-7.

No. 1110 He Tan: Aligning Biomedical Ontologies, 2007 ISBN 978-91-85831-56-2

No. 1112 Jessica Lindblom: Minding the body - Interacting socially through embodied action, 2007 , ISBN 978-91-85831-48-7.

No. 1113 Pontus Wärnestål: Dialogue Behavior Management in Conversational Recommender Systems, 2007 ISBN 978-91-85831-47-0.

No. 1120 Thomas Gustafsson: Management of Real-Time Data Consistency and Transient Overloads in Embedded Systems, 2007, ISBN 978-91-85831-33-3.

No. 1127 Alexandru Andrei: Energy Efficient and Predictable Design of Real-time Embedded Systems, 2007, ISBN 978-91-85831-06-7.

No. 1139 Per Wikberg: Eliciting Knowledge from Experts in Modeling of Complex Systems: Managing Variation and Interactions, 2007, ISBN 978-91-85895-66-3.

No. 1143 Mehdi Amirijoo: QoS Control of Real-Time Data Services under Uncertain Workload, 2007 ISBN 978-91-85895-49-6

No. 1150 Sanny Syberfeldt: Optimistic Replication with Forward Conflict Resolution in Distributed Real-Time Databases, 2007 ISBN 978-91-85895-27-4.

No. 1155 Beatrice Alenljung: Envisioning a Future Decision Support System for Requirements Engineering - A Holistic and Human-centred Perspective, 2008, ISBN 978-91-85895-11-3.

No. 1156 Artur Wilk: Types for XML with Application to Xcerpt, 2008, ISBN 978-91-85895-08-3.

No. 1183 Adrian Pop: Integrated Model-Driven Development Environments for Equation-Based Object-Oriented Languages, 2008, ISBN 978-91-7393-895-2.

No. 1185 Jörgen Skågeby: Gifting Technologies Ethnographic Studies of End-users and Social Media Sharing, 2008, ISBN 978-91-7393-892-1.

No. 1187 Imad-Eldin Ali Abugessaisa: Analytical tools and information-sharing methods supporting road safety organizations, 2008, ISBN 978-91-7393-887-7.

No. 1204 H. Joe Steinhauer: A Representation Scheme for Description and Reconstruction of Object Configurations Based on Qualitative Relations, 2008, ISBN 978-91-7393-823-5.

No. 1222 Anders Larsson: Test Optimization for Core-based System-on-Chip, 2008, ISBN 978-91-7393-768-9.

No. 1238 Andreas Borg: Processes and Models for Capacity Requirements in Telecommunication Systems, 2009, ISBN 978-91-7393-700-9.

No. 1240 Fredrik Heintz: DyKnow: A Stream-Based Know-ledge Processing Middleware Framework, 2009, ISBN 978-91-7393-696-5.

No. 1241 Birgitta Lindström: Testability of Dynamic Real-Time Systems, 2009, ISBN 978-91-7393-695-8.

No. 1244 Eva Blomqvist: Semi-automatic Ontology Construction based on Patterns, 2009 , ISBN 978-91-7393-683-5.

No. 1249 Rogier Woltjer: Functional Modeling of Constraint Management in Aviation Safety and Command and Control, 2009, ISBN 978-91-7393-659-0.

No. 1260 Gianpaolo Conte: Vision-Based Localization and Guidance for Unmanned Aerial Vehicles, 2009, ISBN 978-91-7393-603-3.

No. 1262 AnnMarie Ericsson: Enabling Tool Support for Formal Analysis of ECA Rules, 2009, ISBN 978-91-7393-598-2. 
No. 1266 Jiri Trnka: Exploring Tactical Command and Control: A Role-Playing Simulation Approach, 2009, ISBN 978-91-7393-571-5.

No. 1268 Bahlol Rahimi: Supporting Collaborative Work through ICT - How End-users Think of and Adopt Integrated Health Information Systems, 2009, ISBN 978-91-7393-550-0.

No. 1274 Fredrik Kuivinen: Algorithms and Hardness Results for Some Valued CSPs, 2009 , ISBN 978-91-7393-525-8.

No. 1281 Gunnar Mathiason: Virtual Full Replication for Scalable Distributed Real-Time Databases, 2009 ISBN 978-91-7393-503-6.

No. 1290 Viacheslav Izosimov: Scheduling and Optimization of Fault-Tolerant Distributed Embedded Systems, 2009, ISBN 978-91-7393-482-4.

No. 1294 Johan Thapper: Aspects of a Constraint Optimisation Problem, 2010, ISBN 978-91-7393-464-0.

No. 1306 Susanna Nilsson: Augmentation in the Wild: User Centered Development and Evaluation of Augmented Reality Applications, 2010, ISBN 978-91-7393-416-9.

No. 1313 Christer Thörn: On the Quality of Feature Models, 2010, ISBN 978-91-7393-394-0.

No. 1321 Zhiyuan He: Temperature Aware and Defect-Probability Driven Test Scheduling for System-on-Chip, 2010, ISBN 978-91-7393-378-0.

No. 1333 David Broman: Meta-Languages and Semantics for Equation-Based Modeling and Simulation, 2010, ISBN 978-91-7393-335-3.

No. 1337 Alexander Siemers: Contributions to Modelling and Visualisation of Multibody Systems Simulations with Detailed Contact Analysis, 2010, ISBN 978-91-7393-317-9.

No. 1354 Mikael Asplund: Disconnected Discoveries: Availability Studies in Partitioned Networks, 2010, ISBN 978-91-7393-278-3.

No. 1359 Jana Rambusch: Mind Games Extended: Understanding Gameplay as Situated Activity, 2010, ISBN 978-91-7393-252-3.

No. 1373 Sonia Sangari: Head Movement Correlates to Focus Assignment in Swedish, 2011 ISBN 978-91-7393-154-0.

No. 1374 Jan-Erik Källhammer: Using False Alarms when Developing Automotive Active Safety Systems, 2011 ISBN 978-91-7393-153-3.

No. 1375 Mattias Eriksson: Integrated Code Generation, 2011, ISBN 978-91-7393-147-2

No. 1381 Ola Leifler: Affordances and Constraints of Intelligent Decision Support for Military Command and Control - Three Case Studies of Support Systems, 2011, ISBN 978-91-7393-133-5.

No. 1386 Soheil Samii: Quality-Driven Synthesis and Optimization of Embedded Control Systems, 2011, ISBN 978-91-7393-102-1.

No. 1419 Erik Kuiper: Geographic Routing in Intermittently-connected Mobile Ad Hoc Networks: Algorithms and Performance Models, 2012 ISBN 978-91-7519-981-8.

No. 1451 Sara Stymne: Text Harmonization Strategies for Phrase-Based Statistical Machine Translation, 2012, ISBN 978-91-7519-887-3.

No. 1455 Alberto Montebelli: Modeling the Role of Energy Management in Embodied Cognition, 2012, ISBN 978-91-7519-882-8.

No. 1465 Mohammad Saifullah: Biologically-Based Interactive Neural Network Models for Visual Attention and Object Recognition, 2012, ISBN 978-91-7519-838-5.

No. 1481 David Byers: Improving Software Security by Preventing Known Vulnerabilities, 2012, ISBN 978-91-7519-784-5.

No. 1490 Tomas Bengtsson: Testing and Logic Optimization Techniques for Systems on Chip, 2012,
ISBN 978-91-7519-742-5

No. 1496 Tommy Färnqvist: Exploiting Structure in CSP-related Problems, 2013, ISBN 978-91-7519-711-1.

No. 1503 John Wilander: Contributions to Specification, Implementation, and Execution of Secure Software 2013, ISBN 978-91-7519-681-7.

No. 1506 Magnus Ingmarsson: Creating and Enabling the Useful Service Discovery Experience, 2013, ISBN 978-91-7519-662-6.

No. 1547 Wladimir Schamai: Model-Based Verification of Dynamic System Behavior against Requirements: Method, Language, and Tool, 2013, ISBN 978-91-7519-505-6.

No. 1551 Henrik Svensson: Simulations, 2013, ISBN 978-91-7519-491-2.

No. 1559 Sergiu Rafiliu: Stability of Adaptive Distributed Real-Time Systems with Dynamic Resource Management, 2013, ISBN 978-91-7519-471-4

No. 1581 Usman Dastgeer: Performance-aware Component Composition for GPU-based Systems, 2014, ISBN 978-91-7519-383-0.

No. 1602 Cai Li: Reinforcement Learning of Locomotion based on Central Pattern Generators, 2014, ISBN 978-91-7519-313-7.

No. 1652 Roland Samlaus: An Integrated Development Environment with Enhanced Domain-Specific Interactive Model Validation, 2015, ISBN 978-91-7519-090-7.

No. 1663 Hannes Uppman: On Some Combinatorial Optimization Problems: Algorithms and Complexity, 2015, ISBN 978-91-7519-072-3.

No. 1664 Martin Sjölund: Tools and Methods for Analysis, Debugging, and Performance Improvement of Equation-Based Models, 2015, ISBN 978-91-7519-071-6.

No. 1666 Kristian Stavåker: Contributions to Simulation of Modelica Models on Data-Parallel Multi-Core Architectures, 2015, ISBN 978-91-7519-068-6.

No. 1680 Adrian Lifa: Hardware/Software Codesign of Embedded Systems with Reconfigurable and Heterogeneous Platforms, 2015, ISBN 978-91-7519-040-2

No. 1685 Bogdan Tanasa: Timing Analysis of Distributed Embedded Systems with Stochastic Workload and Reliability Constraints, 2015, ISBN 978-91-7519-022-8.

No. 1691 Håkan Warnquist: Troubleshooting Trucks Automated Planning and Diagnosis, 2015, ISBN 978-91-7685-993-3.

No. 1702 Nima Aghaee: Thermal Issues in Testing of Advanced Systems on Chip, 2015,

ISBN 978-91-7685-949-0.

No. 1715 Maria Vasilevskaya: Security in Embedded Systems: A Model-Based Approach with Risk Metrics, 2015, ISBN 978-91-7685-917-9.

No. 1729 Ke Jiang: Security-Driven Design of Real-Time Embedded System, 2016, ISBN 978-91-7685-884-4

No. 1733 Victor Lagerkvist: Strong Partial Clones and the Complexity of Constraint Satisfaction Problems: Limitations and Applications, 2016, ISBN 978-91-7685-856-1.

No. 1734 Chandan Roy: An Informed System Development Approach to Tropical Cyclone Track and Intensity Forecasting, 2016, ISBN 978-91-7685-854-7.

No. 1746 Amir Aminifar: Analysis, Design, and Optimization of Embedded Control Systems, 2016, ISBN 978-91-7685-826-4

No. 1747 Ekhiotz Vergara: Energy Modelling and Fairness for Efficient Mobile Communication, 2016 , ISBN 978-91-7685-822-6.

No. 1748 Dag Sonntag: Chain Graphs - Interpretations, Expressiveness and Learning Algorithms, 2016, ISBN 978-91-7685-818-9.

No. 1768 Anna Vapen: Web Authentication using 
Third-Parties in Untrusted Environments, 2016, ISBN 978-91-7685-753-3.

No. 1778 Magnus Jandinger: On a Need to Know Basis: A Conceptual and Methodological Framework for Modelling and Analysis of Information Demand in an Enterprise Context, 2016, ISBN 978-91-7685-713-7.

No. 1798 Rahul Hiran: Collaborative Network Security: Targeting Wide-area Routing and Edge-network Attacks, 2016, ISBN 978-91-7685-662-8.

No. 1813 Nicolas Melot: Algorithms and Framework for Energy Efficient Parallel Stream Computing on Many-Core Architectures, 2016, ISBN 978-91-7685-623-9.

No. 1823 Amy Rankin: Making Sense of Adaptations: Resilience in High-Risk Work, 2017, ISBN 978-91-7685-596-6.

No. 1831 Lisa Malmberg: Building Design Capability in the Public Sector: Expanding the Horizons of Development, 2017, ISBN 978-91-7685-585-0.

No. 1851 Marcus Bendtsen: Gated Bayesian Networks, 2017 ISBN 978-91-7685-525-6.

No. 1852 Zlatan Dragisic: Completion of Ontologies and Ontology Networks, 2017, ISBN 978-91-7685-522-5.

No. 1854 Meysam Aghighi: Computational Complexity of some Optimization Problems in Planning, 2017, ISBN 978-91-7685-519-5.

No. 1863 Simon Ståhlberg: Methods for Detecting Unsolvable Planning Instances using Variable Projection, 2017, ISBN 978-91-7685-498-3.

No. 1879 Karl Hammar: Content Ontology Design Patterns: Qualities, Methods, and Tools, 2017, ISBN 978-91-7685-454-9.

No. 1887 Ivan Ukhov: System-Level Analysis and Design under Uncertainty, 2017, ISBN 978-91-7685-426-6.

No. 1891 Valentina Ivanova: Fostering User Involvement in Ontology Alignment and Alignment Evaluation, 2017, ISBN 978-91-7685-403-7.

No. 1902 Vengatanathan Krishnamoorthi: Efficient HTTP-based Adaptive Streaming of Linear and Interactive Videos, 2018, ISBN 978-91-7685-371-9.

No. 1903 Lu Li: Programming Abstractions and Optimization Techniques for GPU-based Heterogeneous Systems, 2018, ISBN 978-91-7685-370-2.

No. 1913 Jonas Rybing: Studying Simulations with Distributed Cognition, 2018, ISBN 978-91-7685-348-1.

No. 1936 Leif Jonsson: Machine Learning-Based Bug Handling in Large-Scale Software Development, 2018, ISBN 978-91-7685-306-1.

No. 1964 Arian Maghazeh: System-Level Design of GPU-Based Embedded Systems, 2018, ISBN 978-91-7685-175-3.

\section{Linköping Studies in Arts and Science}

No. 504 Ing-Marie Jonsson: Social and Emotional Characteristics of Speech-based In-Vehicle Information Systems: Impact on Attitude and Driving Behaviour, 2009, ISBN 978-91-7393-478-7.

No. 586 Fabian Segelström: Stakeholder Engagement for Service Design: How service designers identify and communicate insights, 2013, ISBN 978-91-7519-554-4.

No. 618 Johan Blomkvist: Representing Future Situations of Service: Prototyping in Service Design, 2014, ISBN 978-91-7519-343-4.

No. 620 Marcus Mast: Human-Robot Interaction for Semi-Autonomous Assistive Robots, 2014, ISBN 978-91-7519-319-9.

No. 677 Peter Berggren: Assessing Shared Strategic Understanding, 2016, ISBN 978-91-7685-786-1.

No. 695 Mattias Forsblad: Distributed cognition in home environments: The prospective memory and cognitive practices of older adults, 2016

ISBN 978-91-7685-686-4

\section{Linköping Studies in Statistics}

No. 9 Davood Shahsavani: Computer Experiments Designed to Explore and Approximate Complex Deterministic Models, 2008, ISBN 978-91-7393-976-8.

No. 10 Karl Wahlin: Roadmap for Trend Detection and Assessment of Data Quality, 2008, ISBN 978-91-7393-792-4

No. 11 Oleg Sysoev: Monotonic regression for large multivariate datasets, 2010, ISBN 978-91-7393-412-1.

No. 13 Agné Burauskaite-Harju: Characterizing Temporal Change and Inter-Site Correlations in Daily and Sub-daily Precipitation Extremes, 2011, ISBN 978-91-7393-110-6.

No. 14 Måns Magnusson: Scalable and Efficient Probabilistic Topic Model Inference for Textual Data, 2018, ISBN 978-91-7685-288-0.

\section{Linköping Studies in Information Science}

No. 1 Karin Axelsson: Metodisk systemstrukturering- att skapa samstämmighet mellan informationssystem-arkitektur och verksamhet, 1998, ISBN 9172-19-296-8.

No. 2 Stefan Cronholm: Metodverktyg och användbarhet en studie av datorstödd metodbaserad systemutveckling, 1998, ISBN 9172-19-299-2.

No. 3 Anders Avdic: Användare och utvecklare - om anveckling med kalkylprogram, 1999 ISBN 91-7219-606-8.

No. 4 Owen Eriksson: Kommunikationskvalitet hos informationssystem och affärsprocesser, 2000 ISBN 91-7219-811-7.

No. 5 Mikael Lind: Från system till process - kriterier för processbestämning vid verksamhetsanalys, 2001 ISBN 91-7373-067-X.

No. 6 Ulf Melin: Koordination och informationssystem företag och nätverk, 2002, ISBN 91-7373-278-8.

No. 7 Pär J. Ågerfalk: Information Systems Actability Understanding Information Technology as a Tool for Business Action and Communication, 2003 , ISBN 91-7373-628-7.

No. 8 Ulf Seigerroth: Att förstå och förändra system-utvecklingsverksamheter - en taxonomi för metautveckling, 2003, ISBN 91-7373-736-4.

No. 9 Karin Hedström: Spår av datoriseringens värden Effekter av IT i äldreomsorg, 2004, ISBN 91-7373-963-4.

No. 10 Ewa Braf: Knowledge Demanded for Action Studies on Knowledge Mediation in Organisations, 2004, ISBN 91-85295-47-7.

No. 11 Fredrik Karlsson: Method Configuration method and computerized tool support, 2005 ISBN 91-85297-48-8.

No. 12 Malin Nordström: Styrbar systemförvaltning - Att organisera systemförvaltningsverksamhet med hjälp av effektiva förvaltningsobjekt, 2005 , ISBN 91-85297-60-7.

No. 13 Stefan Holgersson: Yrke: POLIS - Yrkeskunskap, motivation, IT-system och andra förutsättningar för polisarbete, 2005, ISBN 91-85299-43-X.

No. 14 Benneth Christiansson, Marie-Therese Christiansson: Mötet mellan process och komponent - mot ett ramverk för en verksamhetsnära kravspecifikation vid anskaffning av komponent-baserade informationssystem, 2006, ISBN 91-85643-22-X 